\title{
Recent Advances in the Extraction of Polycyclic Aromatic Hydrocarbons from Environmental Samples
}

\author{
Natalia Manousi *(D) and George A. Zachariadis * \\ Laboratory of Analytical Chemistry, Department of Chemistry, Aristotle University of Thessaloniki, \\ 54124 Thessaloniki, Greece \\ * Correspondence: nmanousi@chem.auth.gr (N.M.); zacharia@chem.auth.gr (G.A.Z.); Tel.: +2310-997-707 (N.M.) \\ Academic Editors: Victoria Samanidou and Eleni Deliyanni \\ Received: 15 April 2020; Accepted: 4 May 2020; Published: 7 May 2020 \\ check for \\ updates
}

\begin{abstract}
Polycyclic aromatic hydrocarbons (PAHs) comprise a group of chemical compounds consisting of two or more fused benzene rings. PAHs exhibit hydrophobicity and low water solubility, while some of their members are toxic substances resistant to degradation. Due to their low levels in environmental matrices, a preconcentration step is usually required for their determination. Nowadays, there is a wide variety of sample preparation techniques, including micro-extraction techniques (e.g., solid-phase microextraction and liquid phase microextraction) and miniaturized extraction techniques (e.g., dispersive solid-phase extraction, magnetic solid-phase extraction, stir bar sorptive extraction, fabric phase sorptive extraction etc.). Compared to the conventional sample preparation techniques, these novel techniques show some benefits, including reduced organic solvent consumption, while they are time and cost efficient. A plethora of adsorbents, such as metal-organic frameworks, carbon-based materials and molecularly imprinted polymers, have been successfully coupled with a wide variety of extraction techniques. This review focuses on the recent advances in the extraction techniques of PAHs from environmental matrices, utilizing novel sample preparation approaches and adsorbents.
\end{abstract}

Keywords: PAHs; sample preparation; environmental samples; extraction; MSPE; SPME; FPSE; SBSE; DSPE; PT-SPE.

\section{Introduction}

Polycyclic aromatic hydrocarbons (PAHs) are a large group of chemical compounds composed of two or more fused benzene rings [1]. PAHs are hydrophobic compounds with low water solubility, and their solubility in water and volatility decrease with an increase in their molecular weight [2]. PAHs consisting of up to four rings are known as light PAHs, while PAHs that are made of more than four rings are known as heavy PAHs. Heavy PAHs are more stable and more toxic than the light PAHs. These chemical compounds are widespread environmental contaminants that are considered byproducts of the incomplete combustion of organic materials, such as coal, gas, garbage, meat, oil, tobacco and wood, during natural or anthropogenic processes [1,3]. PAHs are toxic substances, which are resistant to degradation and exposure to them may increase the risk of cancer [4]. As a result, PAHs are considered by US Environmental Protection Agency (EPA) and the European Environmental Agency to be priority pollutants [1]. Therefore, the determination of PAHs in environmental samples is of high importance. Since PAHs exist in traces in environmental matrices, a preconcentration technique is normally required. Figure 1 shows the chemical structures of common PAHs. 


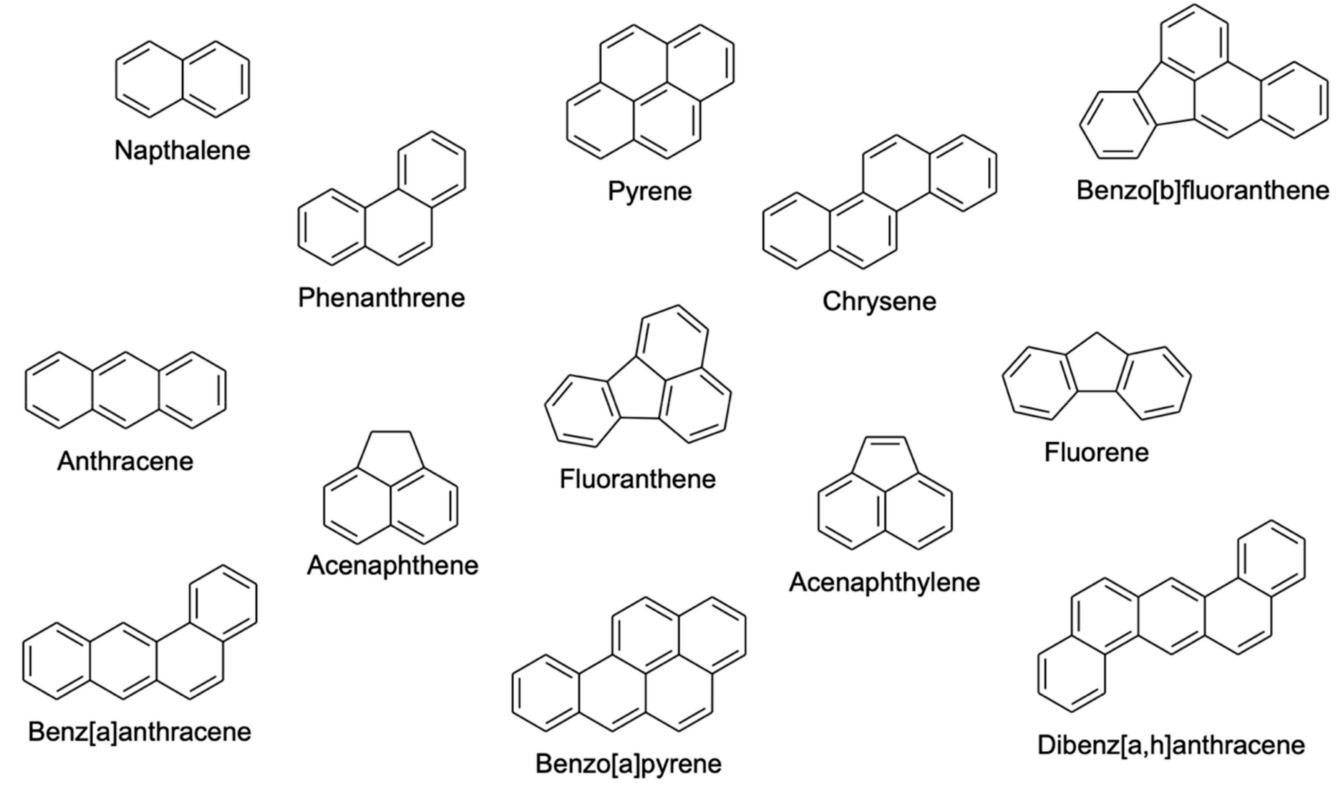

Figure 1. Chemical structures of common polycyclic aromatic hydrocarbons (PAHs).

Currently, the most widely used methods for analyzing these pollutants in environmental matrices are gas chromatography (GC), high performance liquid chromatography (HPLC) and ultra-high pressure liquid chromatography (UHPLC) $[3,5]$. Various detection systems, including ultraviolet detectors (UV) [6], diode array detectors (DAD) [3], fluorescence detectors (FLD) [1], mass detectors (MS) coupled with HPLC and UHPLC and flame ionization detectors (FID) [7], MS detectors [5] and tandem MS detectors (MS/MS) [8] coupled with GC have been used. Due to the enhanced sensitivity in the determination of PAHs that results in lower LODs, mass detectors and tandem MS detectors are generally preferred.

Solid-phase extraction (SPE) and liquid-liquid extraction (LLE) are two major sample preparation techniques that have been widely used for the extraction and preconcentration of a wide variety of analytes from environmental samples. However, both conventional techniques tend to have many fundamental drawbacks, since they include complicated, time-consuming steps, while they require large amounts of sample and organic solvents. Moreover, in both techniques there are difficulties in automation $[9,10]$.

In order to overcome these drawbacks, different microextraction techniques have been proposed as an efficient alternative to classical extraction techniques, since the introduction of solid-phase microextraction (SPME) by the research group of Pawliszyn [11]. Liquid-phase microextraction (LPME) was introduced a few years later by Liu and Dasgupta, by using organic droplets suspended from the tip of a microsyringe [12]. Those microextraction techniques are widely used today, and they offer certain benefits compared to the conventional sample preparation techniques. Microextraction techniques require a significantly lower sample amount, number of extraction steps, sample preparation time and organic solvent consumption, and they comply with Green Analytical Chemistry principles $[10,13]$.

Typical examples of miniaturized sample preparation techniques include dispersive solid phase extraction (d-SPE) [14], magnetic solid-phase extraction (MSPE) [15], pipette tip solid-phase extraction (PT-SPE) [16], fabric phase sorptive extraction (FPSE) [17], stir bar sorptive extraction (SBSE) [18] etc. In recent years, a wide variety of novel sorbents, including molecular imprinted polymers (MIPs), graphene, graphene oxide (GO), carbon nanotubes (CNTs), metal organic frameworks (MOFs), covalent organic frameworks (COFs) and zeolitic imidazole frameworks (ZIFs) have been successfully coupled with miniaturized extraction techniques and microextraction techniques [14,19-21].

Until now, a plethora of publications discuss the different analytical procedures for the extraction of PAHs from liquid, solid and air matrices [22-29]. To the best of our knowledge, in the last five years 
there were no review papers regarding the extraction techniques of PAHs from environmental samples with the novel miniaturized extraction and microextraction techniques. Herein, we aim to discuss the recent advances in the extraction of PAHs from environmental samples. Emphasis will be given to the miniaturized sample preparation approaches, as well as the novel sorbents and other materials that have been successfully coupled with various microextraction techniques.

\section{Extraction of PAHs from Environmental Matrices}

A plethora of novel sample preparation techniques have been employed for the extraction of PAHs. Figure 2 summarizes the recent advances in sorptive extraction techniques that have been employed for the determination of PAHs from environmental samples.

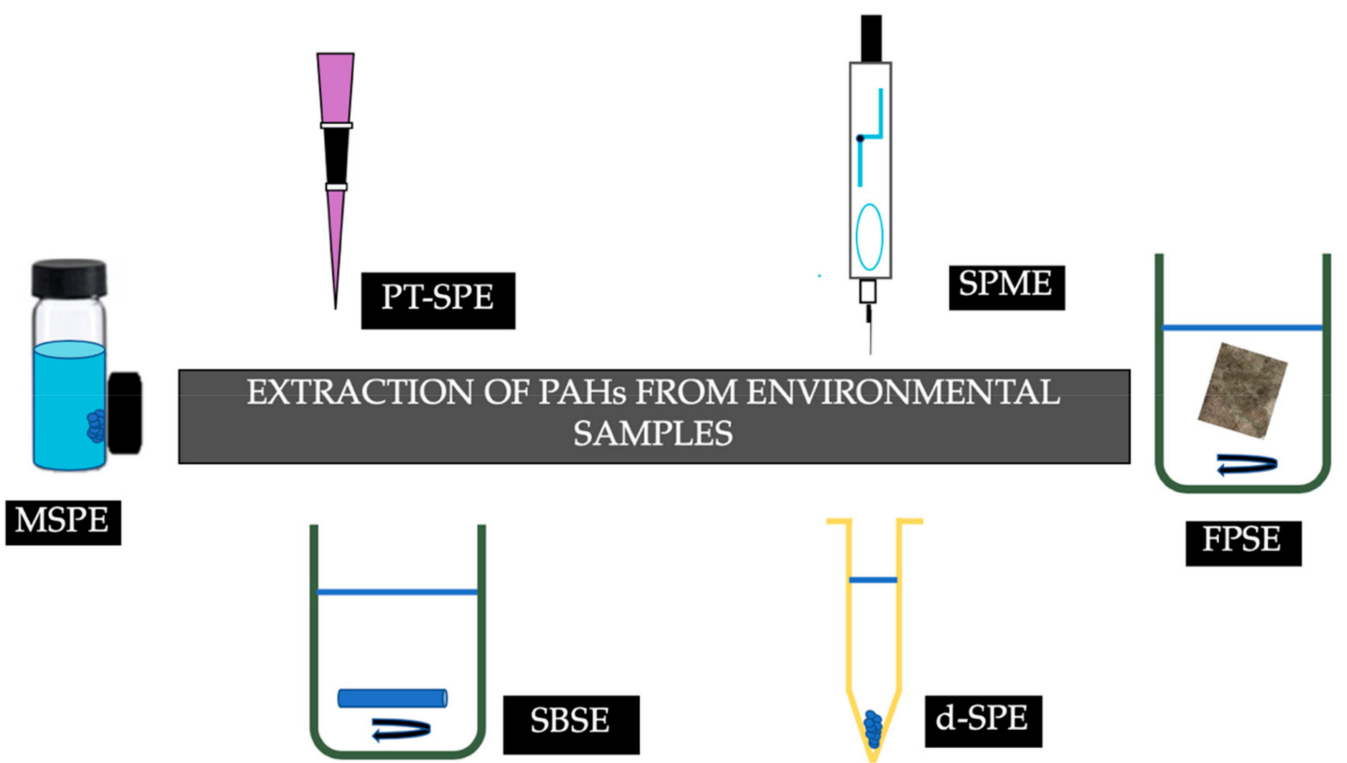

Figure 2. Recent advances in sorptive extraction techniques for the determination of PAHs from environmental samples.

\subsection{Dispersive Solid-Phase Extraction of PAHs from Environmental Matrices.}

Dispersive solid-phase extraction (d-SPE), is a form of SPE in which the desired sorbent is added directly into the sample aqueous solution followed by dispersion. This technique is taking advantage of the contact between the adsorbent and the target analytes. Once the extraction process is completed, the sorbent with the adsorbed analytes is separated from the sample by a mechanical process, such as centrifugation or filtration. Compared to the conventional SPE process, the main benefit of d-SPE is the reduction of sample preparation time, as well as its simplicity, adaptability and easy handling. A wide variety of sorbents have been utilized for the d-SPE of PAHs from environmental samples [30,31].

This technique gained popularity when Anastassiades et al. [32] introduced the QuEChERS (Quick, Easy, Cheap, Effective, Rugged and Safe) approach for the determination of pesticide residue in food of plant origin. The initial method consists of acetonitrile extraction and addition of a mixture of salts, followed by a dispersive clean-up step with a primary-secondary amine (PSA) as extraction sorbent. QuEChERS was quickly applied for the determination of other analytes in a variety of sample matrices. Cvetkovic et al. [33] developed a QuEChERS extraction procedure of PAHs in soil prior to their determination by GC-MS. The researchers evaluated different solvent systems (acetonitrile/water and hexane/water) and sorbents (PSA, $\mathrm{C}_{18}$, Florisil, diatomaceous earth and clinoptilolite). Among the tested parameters, the best results were obtained with acetonitrile/water, as the extraction solvent and diatomaceous earth as the d-SPE extraction adsorbent.

Until today, a wide variety of novel extraction sorbents have been evaluated for the d-SPE of PAHs from environmental matrices. Among them, metal-organic frameworks and zeolitic imidazole 
frameworks are currently the most popular d-SPE adsorbents. MOFs became widely known in 1995, when Yaghi and Li [34] reported the hydrothermal synthesis of a MOF material with large rectangular channels. Metal-organic frameworks are a class of hybrid organic-inorganic supramolecular materials, which are based on the coordination of metal ions or clusters with bi- or multidentate organic linkers. What makes MOFs materials so attractive is their unique properties, such as high surface areas (up to $14,600 \mathrm{~m}^{2} \cdot \mathrm{g}^{-1}$ ) [35], pore size tunability, structure flexibility, luminosity, thermal stability, charge transfer ability from the ligand to the metal or from the metal to the ligands, etc [21,36-39]. As a result, MOFs have gained attention in a plethora of applications, such as gas storage and separation [40], catalysis [41], sensors [42], detoxification [43] and drug delivery [44]. In analytical chemistry, MOFs have been evaluated as stationary phases for GC $[45,46]$ and HPLC $[47,48]$ analysis. However, today, the most popular field of applications of MOFs in analytical chemistry is sample preparation [21,36].

Xia et al. [49] synthesized a JUC-48 (Jilin University China 48) from cadmium nitrate tetrahydrate and biphenyl-4,4'-dicarboxylic acid, and used it for the d-SPE of PAHs from environmental matrices prior to their determination by HPLC-FLD. The novel sorbent exhibited good characteristics regarding its stability and morphology, high surface area and open adsorption site. The researchers observed highly negative relationship between extraction capacity and molecule size. As a result, the JUC-48 adsorbent was used for the extraction of only light PAHs.

Amiri et al. [50] synthesized hybrid nanocomposites prepared from MOF-199 and graphene or fullerene. MOF-199 was prepared from copper nitrate trihydrate and 1,3,5-benzenetricarboxylic acid. Among the examined nanocomposites (i.e., MOF-199, MOF-199/graphene and MOF-199/fullerene), the MOF-199/graphene nanocomposite exhibited the highest adsorption affinity towards PAHs, probably due to the high porosity, surface area and adsorption capacity of graphene.

Zeolitic imidazole frameworks (ZIFs) are a sub-family of metal organic frameworks that favors the benefits of both zeolites and MOFs. ZIFs are composed of imidazolate linkers and metal ions. Their structures are similar to conventional aluminosilicate zeolites. Due to their intrinsic porous characteristics and abundant functionalities, as well as exceptional thermal and chemical stabilities, ZIFs have a wide range of potential applications $[37,51]$.

Liang et al. [52] evaluated the application of a novel cellulose/zeolitic imidazolate frameworks-8 composite for the d-SPE of PAHs from environmental water samples. ZIF-8 is composed of zinc as metal ion and 2-methyl imidazole as an organic linker, and it exhibits good characteristics for the extraction of PAHs due to strong hydrophobic and $\pi-\pi$ interaction, as well as permanent porosity and high surface area. However, ZIF-8 suffers from tiny inner pores and strong hydrophobicity. In order to overcome this drawback, ZIF-8 was modified with cellulose microspheres (CMs) generated from natural cellulose. CMs exhibit macro/mesoporous structures, high surface area and abundance of hydroxyl groups. Due to the combination of the benefits of both ZIF-8 and the CMs, the hybrid material exhibited good extraction characteristics.

Other d-SPE sorbents that have been applied for the extraction of PAHs from environmental samples include graphene/sepiolite [53] and N-acetyl-L-cysteine modified CdS quantum dots [54].

\subsection{Magnetic Solid-Phase Extraction of PAHs from Environmental Matrices.}

Magnetic solid-phase extraction (MSPE) is a form of d-SPE in which a magnetic nanomaterial is added into an aqueous sample solution to adsorb the target analytes. After the adsorption of the analyte, an external magnetic field is applied to collect the sorbent and the supernatant solution is discarded. Subsequently, elution of the adsorbed analytes is achieved with the addition of an appropriate solvent, and magnetic separation is performed once again to collect the eluent, which is further analyzed by a suitable analytical technique. Compared to the conventional SPE procedure, in MSPE there is no need for sorbent packing into cartridges, thus avoiding limitations of column blocking and high pressure. Meanwhile, sample and organic solvent consumption is significantly decreased compared to the classic SPE and LLE formats. Finally, the sorbent separation with a magnet 
is a simple and rapid process, compared to the time-consuming centrifugation and filtration steps that are required in conventional d-SPE [55-57].

Magnetic nanoparticles (MNPs) are characterized by the general formula $\mathrm{MFe}_{2} \mathrm{O}_{4}(\mathrm{M}=\mathrm{Fe}, \mathrm{Co}, \mathrm{Cu}$, $\mathrm{Mn}$, etc.), and they can be produced by a variety of methods, such as co-precipitation, solvothermal, hydrothermal etc. The most common magnetic nanoparticles that have been used in order to fabricate magnetic sorbents for MSPE are $\mathrm{Fe}_{3} \mathrm{O}_{4}$ nanoparticles. Iron oxides have been widely used in MSPE due to their super paramagnetism, their low toxicity, their high magnetic saturation, their simple preparation process and their low price [7]. The application of other magnetic nanoparticles, such as $\mathrm{MnFe}_{2} \mathrm{O}_{4}$, have been also reported [58]. However, the utilization of MNPs in sample preparation has some drawbacks, since their selectivity is low. Moreover, MNPs exhibit low stability in strong acidic aqueous media and low dispersion ability in many sample matrices. Therefore, surface modification of magnetic nanoparticles is usually required to enhance their stability and selectivity by the introduction of special functional groups [7]. As seen in Table 1, a wide variety of chemical compounds including carbon-based materials, polymeric materials, MOFs and other molecules have been employed for this purpose.

\subsubsection{Metal-Organic Framework Magnetic Nanocomposites for the MSPE of PAHs}

A wide variety of metal-organic frameworks have been evaluated as sorbents for the MSPE of PAHs from water samples, after functionalization with magnetic nanoparticles. HKUST-1 (Hong Kong University of Science and Technology), was prepared from copper (II) nitrate hemipentahydrate and benzene-1,3,5-tricarboxylic acid, and has been applied for the MSPE of PAHs from water and fruit tea infusion samples, prior to their determination by UHPLC-FLD. The proposed method provided adequate quantitation and reproducibility of heavy PAHs; however, extraction efficiencies were relatively low and no sorbent reusability was reported [59].

MIL-101(Cr) is another MOF that has been used for the extraction of PAHs after its in situ magnetization [60]. MIL-101 was prepared from chromium nitrate nonahydrate and terephthalic acid, and the magnetic nanocomposite was prepared by the in-situ magnetization of MIL-101 and silica-coated magnetite nanoparticles $\left(\mathrm{Fe}_{3} \mathrm{O}_{4} @ \mathrm{SiO}_{2}\right)$. The novel sorbent exhibited good extraction characteristics, as well as a low sorbent quantity requirement. MIL-101 (Cr) modified zero valent iron nano-particles have been also evaluated as an MSPE adsorbent for the extraction of PAHs [61]. Nanoscale zero valent iron exhibits increased specific surface area and more reactive sites on the surface, compared to $\mathrm{Fe}_{3} \mathrm{O}_{4}$ nanoparticles. Moreover, it is very easy to prepare core-shell $\mathrm{Fe} @ \mathrm{SiO}_{2}$ through a one-step approach under normal temperature and pressure, which directly provides the $\mathrm{SiO}_{2}$ layer. The novel nanocomposite exhibited high extraction efficiency and stability, since it was found to be reusable at least 10 times.

MIL-100(Fe) has been also evaluated as a MSPE sorbent for the extraction of PAHs from water samples [62]. The MIL-100(Fe) sorbent was prepared form iron (III) chloride hexahydrate and 1,3,5-benzenetricarboxylic acid, and it was functionalized with mercaptoacetic acid modified magnetite nanoparticles. The novel sorbent was successfully applied for the MSPE of PAHs from river, well, pond and tap water samples. Although satisfactory extraction recoveries were reported $(>80 \%)$, no data regarding sorbent reusability were provided. Huo et al. [63] prepared a magnetic MIL-100(Fe) nanocomposite through an pyrolytic in situ magnetization approach. In this case, MIL-100 (Fe) was placed in a tube furnace and it was calcined from $300{ }^{\circ} \mathrm{C}$ to $700{ }^{\circ} \mathrm{C}$ under nitrogen. The obtained material exhibited high porosity and magnetic characteristics and it was found to be recyclable and reusable (up to 10 times).

Zhang et al. [64] prepared a zinc benzimidazolate (ZIF-7) nanomaterial anchored onto polydopamine-coated $\mathrm{Fe}_{3} \mathrm{O}_{4}$ nanoparticles. Due to the hydrophobic and $\pi-\pi$ interaction between $\mathrm{ZIF}$ and the target analytes, good extraction efficiency was reported. Moreover, the novel nanocomposite was found to be reusable for at least 10 times. 
Table 1. Application of different sorbents for the magnetic solid-phase extraction (MSPE) of PAHs from environmental samples.

\begin{tabular}{|c|c|c|c|c|c|c|c|c|}
\hline Sorbent & Matrix & $\begin{array}{l}\text { Analytical } \\
\text { Technique }\end{array}$ & $\begin{array}{l}\text { Sorbent Mass } \\
(\mathrm{mg})\end{array}$ & Time (min) & $\begin{array}{l}\text { LODs } \\
\left(\text { ng L L }^{-1}\right)\end{array}$ & $\begin{array}{c}\text { Extraction Recovery } \\
(\%)\end{array}$ & Reusability & Ref. \\
\hline HKUST-1 & Water & UHPLC-FLD & $5 \mathrm{Fe}_{3} \mathrm{O}_{4} / 20$ HKUST-1 & 10 & $0.8-12$ & $39-59$ & NA & [59] \\
\hline MIL-101(Cr) & Water & HPLC-PDA & $1 \mathrm{Fe}_{3} \mathrm{O}_{4} @ \mathrm{SiO}_{2} / 0.6 \mathrm{MIL}-101$ & 20 & $2.8-27.2$ & NA & NA & [60] \\
\hline Fe@MIL-101(Cr) & Water & HPLC-DAD & 50 & 50 & $44-64$ & $>80$ & At least 10 times & [61] \\
\hline MIL-100(Fe) & Water & HPLC-FLD & 10 & 10 & $32-2110$ & $>80$ & NA & [62] \\
\hline MIL-100(Fe) & Water & GC-FID & 12.5 & 15 & $4.6-8.9$ & 73-96 & Up to 10 times & [63] \\
\hline $\mathrm{Fe}_{3} \mathrm{O}_{4} @$ polydopamine/ZIF-7 & Water, particulate matter & GC-MS & $\begin{array}{c}3 \mathrm{Fe}_{3} \mathrm{O}_{4} @ \text { PDA } \\
15 \text { ZIF-7 }\end{array}$ & 40 & $0.71-5.79$ & $>82$ & At least 10 times & [64] \\
\hline TpPa-1 COF & Water & HPLC-FLD & 5 & 21 & $0.24-1.01$ & 73-110 & NA & [65] \\
\hline COF-LZU1@PEI@Fe ${ }_{3} \mathrm{O}_{4}$ & Water, soil & HPLC-FLD & 5 & 33 & $0.2-20$ & NA & At least 6 times & [66] \\
\hline $\mathrm{G} / \mathrm{CNF}$ & Water & GC-FID & 20 & 10 & $4-30$ & $63.0-84.5$ & Up to 6 times & [67] \\
\hline $\mathrm{Fe}_{3} \mathrm{O}_{4} / \mathrm{C}$ & Water & HPLC-FLD & 50 & 30 & $0.2-0.6$ & $76-110$ & At least 10 times & [68] \\
\hline Hydrophilic $\mathrm{Fe}_{3} \mathrm{O}_{4} / \mathrm{C}$ & Water & GC-MS & 10 & 30 & $15-335$ & NA & NA & [5] \\
\hline CNF & Water & GC-FID & 10 & 12 & $8-30$ & NA & At least 10 times & [7] \\
\hline $\mathrm{G} / \mathrm{Fe}_{3} \mathrm{O}_{4} @ \mathrm{PT}$ & Water & GC-FID & 20 & 10 & $9-20$ & $83-107$ & At least 17 times & [69] \\
\hline GO & Water & HPLC-UV & 40 & 16 & 90-190 & $76.8-103.2$ & NA & [6] \\
\hline $\mathrm{GO}-\mathrm{Fe}_{3} \mathrm{O}_{4} @ \mathrm{PS}$ & Water & GC-FID & 15 & 10 & $3-10$ & $69.5-88.7$ & NA & [70] \\
\hline Poly(Py-co-Ani)@GO-Fe ${ }_{3} \mathrm{O}_{4}$ & Water & GC-FID & 35 & & $3-10$ & $50.4-78.3$ & At least 20 times & [71] \\
\hline CNTs & Water & UHPLC-FLD & 5 & 10 & $25-73$ & $76.4-106.5$ & Up to 3 times & [72] \\
\hline mag-MIP & Water & HPLC-PDA & 20 & 55 & $1.3-969$ & $46-100$ & At least 3 times & [73] \\
\hline mag-MIP & Water & GC-MS & $5-20$ & 17 & $30-750$ & $>76$ & NA & [74] \\
\hline RAFT-MIP & Water & GC-MS & 10 & 9 & $1-100$ & $4.5-97$ & NA & [8] \\
\hline PDA & Water & HPLC-FLD & 20 & 5 & $0.5-1.9$ & $76.4-107$ & NA & [75] \\
\hline PPy & Water & GC-MS & 20 & 3 & $0.38-5.01$ & 27.4- 115.7 & NA & [76] \\
\hline PANI/Alginate & Water & HPLC-FLD & 400 & 20 & 10 & $86.0-97.8$ & Up to 6 times & [77] \\
\hline PoT & Water & GC-FID & 60 & 15 & $0.3-5.5$ & NA & Up to 15 times & [58] \\
\hline IL-MNPs & Water & GC-MS & 30 & 8 & $40-1111$ & $75-102$ & Up to 10 times & [78] \\
\hline MNP@CN/IL & Leachate, sludge & HPLC-DAD & 30 & 35 & $400-590$ & $89.50-110.2$ & NA & [79] \\
\hline MNP-PANI-DICAT & Water, sludge, soil & GC-MS & 15 & 40 & $0.8-208.6$ & $80.2-111.9$ & Up to 5 times & [80] \\
\hline $\mathrm{Fe}_{3} \mathrm{O}_{4} @ \mathrm{IL} @ M O$ & Water & HPLC-FLD & 18 & 26 & $0.1-2$ & NA & NA & [81] \\
\hline $\mathrm{Fe}_{3} \mathrm{O}_{4} @ \mathrm{SiO}_{2} @ \mathrm{Nap}$ & Water & HPLC-FLD & 40 & 12 & $0.04-0.12$ & $>90$ & At least 10 times & [1] \\
\hline PC & Water, milk & HPLC-FLD & 100 & 10 & $0.2-0.6$ & $>90$ & NA & [82] \\
\hline $\mathrm{Fe}_{3} \mathrm{O}_{4}-\mathrm{DVB}-\mathrm{SO}_{3}-$ & Water & GC-MS & 50 & 10 & $0.6-2.1$ & $79.9-115.3$ & NA & [83] \\
\hline MPNP & Water & UHPLC-DAD & 200 & 15 & $10.83-18.53 \mathrm{nM}$ & $75.7-106.4$ & At least 5 times & [3] \\
\hline $\mathrm{Fe}_{3} \mathrm{O}_{4} / \mathrm{SiO}_{2} / \mathrm{TPA}$ & Water & HPLC-FLD & 50 & 15 & $0.04-37.5$ & NA & NA & [84] \\
\hline $\mathrm{C}_{18}$ & Water & GC-MS & 50 & 6 & $0.8-36 \times 103$ & $35-99$ & NA & [85] \\
\hline$C_{10}-C_{18}$ carboxylates & Water & HPLC-FLD & 200 & 18 & $0.1-0.25$ & $>90$ & Up to 5 times & [86] \\
\hline n-octadecylphosphonic acid & Water & GC-MS & 50 & 1 & $14.1-70.0 \times 103$ & $61.9-119.1$ & NA & [87] \\
\hline Nylon 6 & Water & HPLC-PDA & 40 & 30 & $0.05-0.58 \times 103$ & $36.2-87.0$ & NA & [88] \\
\hline СТАВ & Water & UHPLC-FLD & $100 \mathrm{Fe}_{3} \mathrm{O}_{4} / 50 \mathrm{CTAB}$ & 30 & $0.4-10.3$ & $59.23-87.95$ & NA & [89] \\
\hline Palm fatty acid & Leachate, sludge & HPLC-DAD & 15 & 25 & $10-50$ & $>81.1$ & Up to 5 times & [90] \\
\hline TBCD & Water & HPLC-FLD & 80 & 15 & $0.03-1.2$ & $>80$ & NA & [91] \\
\hline TCT & Water, urine & HPLC-FLD & 40 & 13 & $0.09-0.15$ & $89-93$ & At least 30 times & [92] \\
\hline $\mathrm{C}_{16}-\mathrm{HO}$ & Water & HPLC-UV & 30 & 24 & $0.14-0.31$ & $88-95$ & Up to 4 times & [93] \\
\hline
\end{tabular}


Covalent organic frameworks (COFs) are novel materials, structurally related to $\mathrm{MOFs}$, consisting of light elements $(\mathrm{H}, \mathrm{O}, \mathrm{C}, \mathrm{N}, \mathrm{B}, \mathrm{Si})$ connected with organic monomers through strong covalent bonds. COFs comprise a class of ordered crystalline porous polymers that are characterized from superior properties, including low crystal density, high specific surface area, pore size tunability and good thermal stability $[36,94,95]$. He et al. [65] fabricated a bouquet-shaped magnetic porous nanocomposite via grafting a COF prepared from 1,3,5-triformylphloroglucinol and p-phenylenediamine (TpPa-1) onto the surface-modified magnetite nanoparticles. The novel sorbent contained clusters of core-shell magnetic nanoparticles and interconnected porous COF nanofibers. Therefore, it exhibited high specific surface area, high porosity, and supermagnetism.

Another covalent organic framework that has been evaluated as adsorbent for PAHs was COF-LZU1 (= Lan Zhou University-1), after immobilization onto polyethyleneimine-functionalized magnetic nanoparticles (COF-LZU1@PEI@ $\mathrm{Fe}_{3} \mathrm{O}_{4}$ ) [66]. For this purpose, the examined COF was prepared from 1,3,5-triformylbenzene and 1,4-diaminobenzene through a Schiff base reaction. The novel sorbent exhibited good stability and reusability. Moreover, due to the strong $\pi$-stacking and hydrophobic interaction, the novel sorbent was successfully applied for the MSPE of PAHs from water and soil samples.

\subsubsection{Carbon-Based Magnetic Sorbents for the MSPE of PAHs}

Magnetic carbon-based nanomaterials exhibit superior extraction characteristics, due to the combination of magnetic nanoparticles with carbon-based materials that pose many interesting properties including good thermal stability and tunable miscibility, as well as good extraction capability [67].

Carbon coated $\mathrm{Fe}_{3} \mathrm{O}_{4}$ nanoparticles were used for the extraction of PAHs from environmental water samples prior to their determination by HPLC-FLD. Due to the large surface area of the nanomaterial and strong adsorption ability of carbon material, high adsorption capacity and extraction efficiency were obtained [68].

A hydrophilic carbon-ferromagnetic nanocomposite was also evaluated for the extraction of PAHs. The sorbent was designed with a hydrophobic sublayer and a hydrophilic surface. As a result, the sorbent exhibited good adsorption efficiency, due to the hydrophobic sublayer of the material, while good compatibility with the aqueous matrices was reported due to its hydrophilic surface [5].

Carbon nanofibers (CNFs) decorated with $\mathrm{Fe}_{3} \mathrm{O}_{4}$ nanoparticles have been also evaluated as adsorbent for the MSPE of PAHs from water samples. Carbon nanofibers are hydrophobic materials with the ability to establish $\pi-\pi$ interaction. Moreover, they exhibit high specific area and flexibility, as well as high mechanical strength [7]. Magnetized graphene $(G)$ layers immobilized on CNFs have been also investigated for the MSPE of PAHs. Graphene is a single layer of carbon atoms that was discovered in 2004 by Geim et al. [96]. In graphene, the carbon atoms are densely packed in a honeycomb crystal lattice, which can be viewed as exfoliated "graphite sheets". Graphene exhibits superior properties, such as a high specific surface area, as well as good chemical stability and thermal stability [6]. The combination of graphene and CNFs provided good adsorption of PAHs that can be attributed to hydrophobic and $\pi-\pi$ interactions [67]. A graphene/ $\mathrm{Fe}_{3} \mathrm{O}_{4} @$ polythiophene $\left(\mathrm{G} / \mathrm{Fe}_{3} \mathrm{O}_{4} @ \mathrm{PT}\right)$ nanocomposite has been also used for the MSPE of PAHs. PT was employed used to enhance the adsorption capacity of magnetic graphene, and also to provide long service life and stability [69].

Graphene oxide is the chemical compound with a form similar to graphene, which consists of one-atom-thick two-dimensional layers of $\mathrm{sp}^{2}$-bonded carbon and is rich in oxygen-containing groups including hydroxyl, carboxyl and epoxy groups. Graphene oxide exhibits high hydrophilicity and dispersibility, as well as good thermal and mechanical stability and high surface area. GO interacts with organic molecules through strong $\pi$-stacking, hydrophobic interaction and hydrogen bonding [57].

Magnetic graphene oxide has been used for the extraction of PAHs from water samples. The composite sorbent exhibited ease in separation and satisfactory recoveries [6]. Polystyrene (PS) modified magnetic $\mathrm{GO}\left(\mathrm{GO}-\mathrm{Fe}_{3} \mathrm{O}_{4} @ \mathrm{PS}\right)$ was fabricated to enhance the extraction efficiency. Polystyrene is an 
inexpensive, high porous, environmentally friendly polymer that contains phenyl and alkyl groups [70]. Another polymeric material that has been employed for the functionalization of magnetic GO is poly(pyrrole-co-aniline) in order to increase its efficiency. Both polyaniline (PANI) and polypyrrole (PPy) are promising materials, due to their good chemical stability and ease of synthesis by the chemical and electrochemical method of polymerization. The PANI and PPy copolymer combines the superior properties of the two conducting polymers and its application for the modification of $\mathrm{Fe}_{3} \mathrm{O}_{4} / \mathrm{GO}$ can result in a highly efficient sorbent [71].

Carbon nanotubes (CNTs) functionalized magnetite nanoparticles have been also employed for the extraction of PAHs from water samples [72]. CNTs are interesting sorbents for volatile and semi-volatile organic compounds due to hydrogen bonding, $\pi$-stacking and hydrophobic interactions. CNTs are divided into single-walled (SWCNTs) and multi-walled carbon nanotubes (MWCNTs), based on whether they consist of one or more sealed tube-shaped layers of graphene, respectively. Adsorption of target analytes can take place in their easily accessible walls, as well as in their interstitial sites [97]. MWCNTs functionalized $\mathrm{Fe}_{3} \mathrm{O}_{4}$ nanoparticles showed good extraction efficiency and selectivity towards PAHs [72].

\subsubsection{Molecularly Imprinted Polymers Magnetic Nanocomposites for the MSPE of PAHs}

Molecularly Imprinted Polymers (MIPs) are synthetic polymeric materials that are composed of imprinted sites complementary to a specific chemical molecule. MIPs show high affinity towards the target analytes with analogous molecular structure, resulting in high extraction ability [55]. Molecular recognition is attributed to a combination of shape and size and hydrogen bonding, as well as electrostatic and hydrophobic interactions. MIPs are stable at a wide $\mathrm{pH}$ and temperature ranges and in most organic solvents, while there is normally no requirement for special storage conditions $[98,99]$.

MIPs can be prepared through covalent, non-covalent and semi-covalent approaches, and their preparation is based on the polymerization of a functional monomer and a cross-linker around a template molecule. The template molecule should be able to interact with the functional monomer to develop complexes that further interact with the cross-linker during the polymerization reaction. Subsequently, the template molecule is removed and a MIP with imprinted sites complementary to the molecular structure and the functional groups of the template is created. Normally, after the synthesis of MIPs, extensive washing is required to remove any residual molecules of template. However, even after many washing steps, bleeding of the template has been reported. In order to overcome this drawback, MIPs can be prepared from templates with chemical structure that is analogue to the target molecules $[98,100]$.

Magnetic MIPs combine the benefits of ease in separation of magnetic materials with the high molecular recognition of MIPs. Many researchers reported the synthesis of magnetic MIPs as adsorbents of PAHs from environmental samples.

Villar-Navarro et al. [73] used a commercially available magnetic MIP (mag-MIP) by NanoMyP ${ }^{\circledR}$ (Granada, Spain) that was able to extract the 16 EPA-regulatd PAHs. The examined sorbent exhibited good extraction efficiencies (98.8-100\%) for the more lipophilic PAHs, however, for the less lipophilic PAHs, extraction recovery rates were lower (46-60\%). Finally, mag-MIP was found to be reusable at least three times. Benedetti et al. [74] managed to improve the extraction efficiencies by implementing a Plackett-Burman experimental design. In this case, all recovery values were higher than $76 \%$.

Azizi et al. [8] reported the synthesis of a MIP sorbent by surface polymerization onto magnetic $\mathrm{Fe}_{3} \mathrm{O}_{4} @ \mathrm{SiO}_{2}$ nanoparticles through reversible addition fragmentation chain transfer (RAFT) polymerization. For this purpose, methacrylic acid and isopropyl acrylamide were used as functional monomers. In this case, extraction recoveries ranged from $4.5 \%$ to $97 \%$, thus extraction of "heavy" PAHs was found to be more efficient compared with the extraction of "lighter" PAHs. 


\subsubsection{Polymer-Modified Magnetic Nanoparticles for the MSPE of PAHs}

Polymers are promising materials in separation science, due to the fact that their network can be chemically anchored around the magnetic core, thus providing large $\pi$-conjugated structure, hydrophobicity and polar functional groups for adsorption [58]. Various polymers including polydopamine [75], polypyrrole [76], poly(o-toluidine) [58] and polyaniline [77]. Polyaniline and its derivatives are one of the widely used polymers for the extraction of various types of organic compounds, because of their good stability in high temperature, air and different solvents [58].

Polydopamine (PDA) functionalized magnetic nanoparticles have been also employed for the extraction of PAHs. PDA exhibit several significant advantages, including biocompatibility, good dispersibility in water, as well as multifunctional groups (amino and catechol groups) that enhance the extraction efficiency of PAHs due to $\pi$-stacking interaction [75].

Polypopyrole [76], due to its hydrophobicity, large $\pi$-conjugated structure, hydrogen bonding and ion exchange properties, exhibits good extraction efficiency and selectivity towards PAHs.

Polyaniline-coated magnetite nanoparticles incorporated in alginate beads have also been employed for the MSPE of PAHs in water samples. In this case, the alginate beads assisted in the increase of the surface area for polyaniline coating, and the novel nanocomposite showed satisfactory extraction efficiency towards the PAH analytes, due to the $\pi-\pi$ interactions between the polyaniline moieties [77].

Poly(o-toluidine) (PoT) coated $\mathrm{MnFe}_{2} \mathrm{O}_{4}$ magnetic nanoparticles have been also successfully employed as a magnetic adsorbent for the MSPE of PAHs from water samples [58]. Due to the $\pi-\pi$ interactions between the active sites of PoT and PAHs, a satisfactory extraction efficiency was reported.

\subsubsection{Ionic liquids Modified Magnetic Nanoparticles for the MSPE of PAHs}

Ionic liquids (ILs) are a green alternative to conventional organic solvents that have gained a lot of attention lately. ILs are generally composed of bulky, non-symmetrical organic cations (i.e., imidazolium, pyrrolidinium, pyridinium, ammonium, phosphonium etc.) and different inorganic or organic anions (i.e., tetrafluoroborate anions, bromide anions etc.) [101-103]. The increased popularity of ILs may be attributed to their extraordinary properties, such as a negligible vapor pressure, good thermal stability, as well as tunable viscosity and miscibility with water and organic solvents. By choosing the cationic or the anionic constituent of ILs, their polarity, hydrophobicity, viscosity and other chemical and physical properties can be tuned, in order to prepare ILs with the desired characteristics. Due to their special structures, ionic liquids also exhibit good extractability for various organic compounds and metal ions [103]. As a result, ionic liquids have been successfully coupled with various extraction techniques for the extraction of PAHs.

Galán-Cano et al. [78] prepared ionic liquid-coated magnetic nanoparticles (IL-MNP) and used them for the MSPE of PAHs from water samples, prior to their determination by GC-MS. For this purpose, methylimidazolium-chloride was used to modify $\mathrm{Fe}_{3} \mathrm{O}_{4} @ \mathrm{SiO}_{2}$ magnetic nanoparticles by a simple metathesis reaction, using potassium hexafluorophosphate as a reagent. Due to the covalent immobilization of the ionic liquid onto the surface of silica coated $\mathrm{Fe}_{3} \mathrm{O}_{4}$ nanoparticles, the developed sorbent exhibited good stability, and it was found to be reusable for up to 10 times.

Cyano-ionic liquid functionalized magnetic nanoparticles (MNP@CN/IL) have been also employed for the MSPE of PAHs from environmental water samples, prior to their determination by HPLC-DAD [79]. For this purpose, 1-benzyl-3-(trimethoxysilylpropyl)imidazolium chloride was chosen as IL, and it was used to modify the cyano-functionalized magnetic nanoparticle. The combination of the cyano group and IL on the surface of the MNPs provided good extraction efficiency, probably due to the combination of $\pi-\pi$ and electrostatic interaction.

Magnetic nanoparticles coated with a polyaniline-di-cationic ionic liquid (MNP-PANI-DICAT) were prepared and used for the MSPE of PAHs in environmental samples [80]. The novel sorbent combined the benefits of PANI and ionic liquids. Due to the $\pi-\pi$ interaction between polyaniline shell 
and di-cationic IL with PAHs compounds, high extraction efficiency was observed. Moreover, the novel MSPE sorbent exhibited satisfactory stability, since it was found to be reusable up to five times.

Liu et al. [81] fabricated a magnetic ionic liquid functionalized methyl orange nanoparticles ( $\left.\mathrm{Fe}_{3} \mathrm{O}_{4} @ \mathrm{IL} @ \mathrm{MO}\right)$, by self-assembly of the 1-octadecyl-3-methylimidazolium bromide and methyl orange on the surface of $\mathrm{Fe}_{3} \mathrm{O}_{4}$ silica magnetic nanoparticles. The novel sorbent was successfully applied for the MSPE of PAHs from environmental water samples. Due to the presence of benzene rings of methyl orange and the hydrocarbon chains of ILs, the sorbent provided adsorption sites for organic pollutants through $\pi-\pi$ and hydrophobic interactions. The silica modified $\mathrm{Fe}_{3} \mathrm{O}_{4}$ nanoparticles were found to be reusable after washing, however, assembling with IL and methyl orange was also required.

\subsubsection{Other Magnetic Nanocomposites for the MSPE of PAHs}

Naphthyl functionalized magnetic nanoparticles $\left(\mathrm{Fe}_{3} \mathrm{O}_{4} @ \mathrm{SiO}_{2} @ \mathrm{Nap}\right)$ have been used for the extraction of PAHs from river waters, prior to their determination by HPLC-FLD. Due to the condensed cyclic structure and the hydrophobic property of naphthyl, the novel sorbent exhibited satisfactory selectivity and extraction efficiency through $\pi-\pi$ interactions [1].

Phosphatidylcholine (PC) is a phospholipid consisting of a long double carbon chain, and the zwitterions' pair headgroup is composed of phosphate and choline. The extraction of PAHs can be assisted, due to the middle hydrocarbon chains that provide adsorption sites due to hydrophobic interactions. Meanwhile, the zwitterions pair headgroups endow the outer surface of the substrate with perfect hydrophilicity and biocompatibility [82].

Another example of composite material exhibiting both hydrophilicity and hydrophobicity is the magnetic nanocomposite prepared from nanoparticles functionalized with divinylbenzene (DVB) and sulfonate moieties $\left(\mathrm{Fe}_{3} \mathrm{O}_{4}-\mathrm{DVB}-\mathrm{SO}_{3}{ }^{-}\right)$. In this case, the hydrophobic DVB moieties were dedicated for extraction, while the hydrophilic sulfonate groups were introduced to enhance dispersion of the magnetic sorbent in the aqueous sample solution [83]. A hydrophilic-hydrophobic magnetic $\mathrm{Fe}_{3} \mathrm{O}_{4}$-doped polymeric nanoparticle (MPNP), prepared from highly charged poly(styrene-divinylbenzene-co-4-vinylbenzenesulfonic acid sodium salt) has been also employed for the determination of PAHs in environmental matrices [3].

Triphenylamine (TPA) has been employed for the functionalization of magnetic nanoparticles $\left(\mathrm{Fe}_{3} \mathrm{O}_{4} / \mathrm{SiO}_{2} / \mathrm{TPA}\right)$. Due to the strong $\pi-\pi$ conjugate effect between the three benzene rings of TPA and PAHs, satisfactory extraction efficiency and selectivity were observed [84].

Other examples of chemical compounds and groups that have been employed for the functionalization of magnetic nanoparticle for the MSPE of PAHs from environmental samples are $C_{18}$ [85], hemimicelles of alkyl $\left(C_{10}-C_{18}\right)$ carboxylates [86], n-octadecylphosphonic acid [87], nylon 6 [88], cetyltrimethylammonium bromide (CTAB) [89], palm fatty acid [90], 1,4,7,10-tetrabenzyl-1,4,7,10-tetraazacyclododecane (TBCD) [91], tetraazacalix[2]arence[2]triazine (TCT) [92] and $n$-hexadecylsilanol-diol $\left(\mathrm{C}_{16}-\mathrm{HO}\right)$ [93].

\subsection{Solid-Phase Microextraction of PAHs from Environmental Matrices.}

Solid-phase microextraction is a sample preparation microextraction technique in which the analytes are directly extracted and preconcentrated at the outer coating of a fused-silica fiber [104]. There are two approaches of SPME that can be used to extract analytes, the headspace (HS-SPME), where the fiber is exposed to the gas phase above the sample, and direct immersion (DI-SPME), where the fiber is directly immersed into the sample solution [105]. After the extraction, desorption takes place either thermally in the injection port of a gas chromatograph or by the addition of an organic solvent $[104,105]$.

Until now, there are many different commercial SPME coated fibers, such as polydimethylsiloxane (PDMS), polydimethylsiloxane/divinylbenzene (PDMS/DVB), polyacrylate (PA), carboxen/polydimethylsiloxane(CAR/PDMS) and divinylbenzene/carboxen/polydimethylsiloxane (DVB/CAR/PDMS) [105]. However, most of them more or less have some disadvantages, such as 
low selectivity, ease of fiber breakage, a short lifetime and swelling in organic solvents. In order to overcome them, various new coatings have been prepared and evaluated [106].

Ionic liquids and polymeric ionic liquids (PILs) have been successfully employed as SPME coatings, due to their simplicity of synthesis and their high tuneability, that enable the preparation of highly selective fibers [107]. PILs are polymers prepared from IL monomers. Compared with conventional ILs, PILs exhibit a number of advantages when used as coatings in SPME. Generally, PILs often have solid nature and good thermal and mechanical strength, while extraction selectivity is similar with ILs. As a result, they have proved to be more stable coatings $[107,108]$. Various PILs including poly(1-vinyl-3-hexadecylimidazolium) bis[(trifluoromethyl)sulfonyl]imide [109], poly(1-4-vinylbenzyl)-3-hexadecylimidazolium bis[(trifluoromethyl)sulfonyl]imide [110], poly (1-vinyl-3-octylimidazolium) 2-naphthalene-sulfonate [111] and poly(1-(4-vinylbenzyl)-3-hexadecylimidazolium bis[(trifluoromethyl)sulfonyl]imide [112] have been successfully used as coatings used for the SPME of PAHs. In most cases, the PIL was initially prepared and diluted in a volatile solvent (i.e., acetone or chloroform), and a bare fiber (usually made from stainless steel) was immersed in the solution, followed by slow removal and air drying to remove any excess of solvent that may contribute to high background signals in gas chromatography $[109,110,112]$. However, in situ polymerization of the IL and creation of the SPME coating on the surface of a stainless steel wire has also been reported [111].

Graphene [113,114], graphene oxide [115-117], MWCNTs [118,119] and other carbon based materials [120] have also been successfully employed as SPME sorbent coatings, either neat or combined with other materials in order to generate more efficient composites. These materials exhibit high chemical, thermal and mechanical stability, as well as great affinity towards PAHs. Additionally, due to their unique structures and sufficient surface areas, rapid extraction and desorption of the target analytes can be achieved [107]. Various techniques for the preparation of the coated fibers including the chemical bonding [113,115,117], electrophoretic deposition [119] and sol-gel approaches [120] have been also evaluated.

Metal-organic frameworks (MOFs) are also promising sorbents for SPME coatings $[107,121]$. A wide variety of MOF materials, such as HKUST-1 [122], MOF-199 [123], ZIF-8 [124], TMU-6 (Tarbiat Modares University) prepared from $\mathrm{N}^{1}, \mathrm{~N}^{4}$-bis((pyridin-4-yl)methylene)-benzene-1,4-diamine and 4,4'-oxybisbenzoic acid) [125], MOF-177 prepared from zinc nitrate hexahydrate and 1,3,5-tris(4-carboxyphenyl)-benzene [126], UiO-6 (University of Oslo) prepared from zirconium chloride and terephthalic acid [127], an ytterbium based MOF prepared from aminoisophthalic acid and 2,2'-bipyridine [128], as well as bio-MOF-1 prepared from 4,4'-biphenyl dicarboxylic acid (BPDC), zinc acetate dihydrate and adenine [129].

The main considerations when preparing MOFs for extraction purposes are their stability under extraction conditions, and their ability to establish interactions with the target analytes. Moreover, when fabricating MOFs as coatings for SPME fibers the mechanical and thermal stability should be carefully evaluated. The main procedures that can be used to obtain MOF coatings include physical adhesion, immersion in a suspension of MOF, in situ growth and electrodeposition [107].

A metal-azolate framework 66 (MAF-66) has been also used for the SPME of PAHs from environmental samples. Metal-azolate frameworks are a subfamily of MOFs that have recently gained attention. MAF-66 was prepared from 3-amino-1,2,4-triazole and zinc hydroxide and was used to fabricate a SPME coating by a layer-by-layer deposition method, providing enhancement factors of 123-3108 [130].

Examples of other materials that have been employed to fabricate SPME coatings for the extraction of PAHs include polyaminithiophenol (PATP) with Au coating [131], poly(3,4-ethylenedioxythiophene)@gold nanoparticles[132], crosslinked methyl methacrylate-polyhedral oligomeric silsesquioxane hybrid polymeric coating [106], nanostructured octadecyl silica [133] and polythiophene/carboxylic acid modified multi-walled carbon nanotube 
composite [134]. In the latter approach, the researchers developed a novel SPME technology, in which the features of heating the sample, cooling the sorbent and extraction under vacuum condition were combined [134].

PAL (Prep And Load solution) SPME Arrow technique [135] has been also evaluated for the extraction of PAHs from environmental samples. This technique is based on the use of a robust stainless-steel backbone, carrying the connection to the PAL sampler, the coating and an arrow-shaped tip for septum penetration. SPME Arrow combines the benefits of conventional SPME with the larger sorption phase volumes that are used in stir bar sorptive extraction (SBSE). At the same time, the disadvantages of both techniques include the difficulty in automation for SBSE, and the small volume of sorption phase, as well as the low robustness of classical SPME fibers. The results indicated that extraction efficiency significantly benefited from the larger sorption phase volume.

\subsection{Stir Bar Sorptive Extraction (SBSE) and Stir Rod Sorptive Extraction (SRSE) of PAHs from Environmental Matrices.}

Stir bar sorptive extraction was initially introduced by Baltussen et al. in 1999 [136]. In SBSE, a coated stir bar is placed into the vial together with the aqueous sample solution. Extraction of the target analytes takes place under rigorous stirring. When equilibrium is reached, the stir bar is removed and elution of the adsorbed analytes takes place either by the addition of an organic solvent or thermally [18]. SBSE by nature is an equilibrium technique, and for water samples the extraction of the target analytes into the extraction medium is controlled by the partitioning coefficient of the solutes between the coating phase and the aqueous phase [137]. Polydimethylsiloxane (PDMS) is the most used commercially available coating phase for stir bars, however, the synthesis and application of many novel coating materials has been reported.

PDMS coated SBSE bars have been successfully used for the extraction of PAHs resulting in good recoveries and low detection limits [138-142]. Apart from the conventional PDMS stir bars, various novel coating materials have been evaluated for the extraction of PAHs from environmental samples. Typical examples are polymeric materials that have been evaluated, as stir bar coatings are polypyrrole and polyaniline copolymer (PPy-PAN) [143] and (octyl methacrylate- ethylene dimethacrylate) copolymer [144]. Poly (ethylene glycol)-grafted multi-walled carbon nanotubes have been also evaluated for the extraction of PAHs from environmental samples [145]. In this case, the extraction efficiency was favored by the superior characteristics of MWCNTs and the ease in operation of the SBSE technique.

$\mathrm{Hu}$ et al. [146] prepared polydimethylsiloxane/metal-organic framework (PDMS/Al-MIL-53-NH2) coated stir bars and used them for the extraction of PAHs. The novel PDMS/MOFs-coated stir bars achieved higher extraction efficiencies for PAHs than the commercial PDMS-coated stir bar. In this case, the reported MOF was prepared from terephthalic acid and aluminum chloride. In contrast to the conventional PDMS coating, in which extraction is based only on hydrophobic interactions, the PDMS/MOF composite also takes advantage of the $\pi-\pi$ conjugations.

Benede et al. [147] used stir bar dispersive liquid microextraction (SBDLME) for the extraction of PAHs from natural waters. SBDLME is a hybrid approach that combines the benefits of SBSE and DLLME. For this purpose, a neodymium stir bar was magnetically coated with a magnetic ionic liquid. At high stirring rates, the ionic liquid was dispersed into the solution, followed by being magnetically retrieved onto the stir bar when the extraction was completed. Subsequently, thermal desorption took place and PAHs were determined in a GC-MS system. The novel method exhibited high sensitivity and low LOD values.

Luo et al. [148] developed a stir rod sorptive extraction (SRSE) method for the preconcentration PAHs from water samples, prior to their determination by GC-MS. SRSE is an improved format of SBSE, which has been proposed to avoid the friction loss of extraction coatings that exist in conventional SBSE procedure. In SBSE, a stir rod (glass insert with coating) is immersed in the sample solution to adsorb the target analytes [149]. For the extraction of PAHs, a graphene-polymer composite was 
evaluated. Due to the high specific surface area and $\pi$-stacking properties of the hybrid coating, good extraction efficiency was reported [148].

Automated stir plate sorptive extraction (SPSE) coupled with HPLC-FLD has been also evaluated for the extraction of PAHs. For this purpose, automatic extraction, desorption and sample loading, was controlled by a programmable flow injection system, and extraction of PAHs took place on the surface of a PDMS/ $\beta$-cyclodextrin/divinylbenzene (PDMS/ $\beta-C D / D V B)$ coated plates. The researchers investigated three different operation modes, including static, circular flow and continuous flow SPSE. It was found that extraction efficiencies with continuous flow SPSE were slightly better than circular and manual SBSE, probably due to the continuous introduction of new sample solutions [150].

\subsection{Liquid-Phase Microextraction of PAHs from Environmental Matrices.}

Liquid-phase microextraction (LPME) is a miniaturized version of classical LLE, which is characterized by minimum consumption of solvents. LPME can be divided into three main categories, the single-drop microextraction (SDME), the hollow fiber liquid-phase microextraction (HF-LPME) and the dispersive liquid-liquid microextraction (DLLME), with the latter being the most widely used LPME form [151].

HF-LPME is usually based on the use of disposable propylene porous hollow fibers that are filled with a small amount of extracting solvent (acceptor phase). In order to extract the target analytes, the fibers are immersed into the aqueous sample solution (donor phase) [151,152].

On the other hand, in single-drop microextraction (SDME), a drop acts as the acceptor phase for the extraction. SDME can be divided into two main categories i.e., the direct-immersion single-drop microextraction (DI-SDME), in which the drop is directly immersed into the sample, and the headspace single-drop microextraction (HS-SDME), in which the drop is suspending over the sample [153].

DLLME is based on the initial fast injection of a suitable mixture of two solvents, an extraction solvent and a dispersive solvent, into an aqueous sample solution with the assistance of a syringe, followed by the formation of a cloudy solution that contains droplets of the extraction solvent dispersed into the sample. After phase separation due to the difference in density of the two phases (e.g., by centrifugation), the extraction phase can be removed and analyzed. In the conventional form of DLLME, the extraction phase is accumulated at the bottom of the extraction container [151,154]. DLLME is considered to be simple, cheap and environmentally friendly, while it provides high enrichment. The proper selection of the extraction and dispersive solvents are two critical factors for the optimization of DLLME procedure. Therefore, the dispersive solvent has to be immiscible with the extraction solvent and the aqueous sample, in order to generate a cloudy solution that increases the interaction between the two phases, in order to increase the extraction efficiency [155].

Until today, many different DLLME methods have been proposed for the extraction of PAHs. In 2006, Rezaee et al. [156] developed a DLLME method for the determination of PAHs in surface water, using tetrachloroethylene and acetone as extraction and disperser solvent, respectively. In order to decrease the extraction time, the main drawback of DLLME is the requirement for organic solvents with a density higher than water, in order to be sedimented at the centrifugation step. Guo et al. [157] proposed a low-density solvent-based solvent demulsification DLLME approach. For this purpose, after the formation of the emulsion of the aqueous sample, the disperser solvent (acetone) and the extraction solvent ( $n$-hexane), more acetone was added as demulsification solvent, to break up the emulsion. Since no centrifugation was required, the whole procedure was very rapid (2-3 min). A special extraction cell has been also employed for the DLLME of PAHs, based on the use of low-density solvent. Hosseini et al. [158] evaluated the application of air flotation to assist the phase separation, using toluene as an extraction solvent. In this case, the organic solvent was collected at the top part of the designed cell, and no centrifugation was required in this procedure.

The combination of ultra-sound radiation [159] and vortex radiation [160] with DLLME has been evaluated in order to assist the extraction process, and to achieve high extraction efficiency in a short period of time [161]. With the assistance of radiation, the mass transfer of target analytes from 
the sample to the extraction solvent was facilitated, due to the shorter diffusion distance and larger interfacial area [160].

Ultrasound-assisted emulsification microextraction (USAEME) is a similar approach with ultra-sound assisted DLLME, however, in USUAEME, the dispersion of the extraction solvent in the aqueous sample is attributed to ultra-sound radiation, and no disperser solvent is added. In this case, ultrasonic radiation is used to overcome the drawbacks of disperser solvent, such as the decrease of partition coefficient of analytes into an extraction solvent al [161,162]. USAEME methods for the extraction of PAHs from environmental samples, using toluene [161], chloroform [162], iso-octane [163] and cyclohexane [164] as extraction solvents have been reported. The combination of ultrasound radiation and iso-octane as extraction solvent enabled the handling of high volumes of sample, thus, after concentrating the organic solvent phase, enhancement factors of up to 100,000 were reported. Moreover, the phase separation of iso-octane and sample was assisted by the addition of sodium chloride ( $\mathrm{NaCl})$, and no centrifugation was required [163].

An interesting approach to DLLME and USAEME that can be employed for the separation of the extraction solvent is the solidification of floating organic droplet (SFO). In this case, the sample solution is transferred into an ice bath after the extraction process and the floating organic droplet is allowed to solidify. When solidified, the droplet is removed and placed in a vessel to melt prior to the instrumental analysis $[165,166]$. Yousefi et al. [167] evaluated the use of a deep eutectic solvent (DES) tetra-n-butyl ammonium bromide (TBAB) carboxylic acids for the extraction of PAHs from water samples based on solidification of floating organic droplet. DESs are eco-friendly solvents, which are usually prepared from a hydrogen bond acceptor (e.g., choline chloride salt) and a hydrogen bond donor (e.g., urea, glycerol, carboxylic acids etc.) that can be associated with each other with hydrogen bond interactions. Among the benefits of DESs is their easy preparation from biocompatible, nontoxic and biodegradable chemical compounds $[167,168]$.

The application of ionic liquids in DLLME has been also studied in order to replace the environmentally hazardous volatile organic solvents that are commonly used. Pena et al. [169] developed a DLLME procedure using 1-octyl-3-methylimidazolium hexafluorophosphate $\left(\left[\mathrm{C}_{8}\right.\right.$ $\left.\mathrm{MiM}]\left[\mathrm{PF}_{6}\right]\right)$ IL as extraction solvent. Due to the chemical affinity between the IL and the PAHs, good extraction recoveries and enhancement factors was reported.

In order to enhance the extraction efficiency and the enhancement factors, Liu et al. [170] combined an SPE method based on a macrocyclic polyamine-functionalized silica with an ionic liquid DLLME procedure. For this purpose, after the elution of PAHs from the SPE column with acetone, distilled water and 1,3-dibutylimidazolium bis[(trifluoromethyl)sulfonyl]imide ([BBIM][Tf $\left.\left.{ }_{2} \mathrm{~N}\right]\right)$ as extraction solvents were rapidly injected for the DLLME procedure.

In order to enhance the sensitivity of the determination of PAHs in environmental matrices, Shamsipur and Hashemi [171] combined the SBSE with dispersive liquid-liquid microextraction, based on the solidification of floating organic drop. For this purpose, the target analytes were extracted on the surface of a PDMS coated stir bar, which was further placed in a glass vial containing methanol (disperser solvent). After the elution of PAHs, the stir bar was removed and 1-undecanol was added as an extraction solvent. After centrifugation and cooling in an ice bath, the organic drop was collected. Finally, the drop was again melted at room temperature, mixed with acetonitrile and analyzed in a HPLC-UV system. The combination of the extraction techniques provided good extraction recoveries and low LOD values.

Finally, Fernandez et al. [172] developed a lab on valve DLLME method for the extraction of PAHs, prior to their determination by HPLC-FLD. For this purpose, trichloroethylene was used as the extraction solvent, and acetonitrile was used as the dispersive solvent. The automated instrumentation simplified the extraction process and exhibited satisfactory enhancement factors (86-95). Table 2 summarizes the applications of DLLME and USAEME in the extraction of PAHs from water samples. Even though DLLME is the predominant form of LPME that has been employed for the extraction of PAHs, the use of SDME $[173,174]$ and HF-LPME $[175,176]$ has been also evaluated. 
Table 2. Applications of dispersive liquid-liquid microextraction (DLLME) and ultrasound-assisted emulsification microextraction (USAEME) in the extraction of PAHs from water samples.

\begin{tabular}{|c|c|c|c|c|c|c|c|c|}
\hline Matrix & $\begin{array}{l}\text { Analytical } \\
\text { Technique }\end{array}$ & Extraction Solvent & $\begin{array}{c}\text { Disperser } \\
\text { Solvent }\end{array}$ & Phase Separation & $\operatorname{LODs}\left(\mathrm{ng} \cdot \mathrm{L}^{-1}\right)$ & EF & $\begin{array}{c}\text { Extraction } \\
\text { Recovery (\%) }\end{array}$ & Ref. \\
\hline Surface water & GC-MS & Tetrachloroethylene & Acetone & Centrifugation & $7-30$ & $603-1113$ & - & [156] \\
\hline Rainwater & GC-MS & $n$-Hexane & Acetone & $\begin{array}{c}\text { Addition of } \\
\text { demulsification solvent }\end{array}$ & $3.7-39.1$ & NA & - & [157] \\
\hline River water & GC-FID & Toluene & Methanol & Air flotation & $14-41 \times 10^{3}$ & NA & - & [158] \\
\hline Sea water & GC-MS & Tetrachloroethylene & Diethyl Ether & Centrifugation & $1-10$ & $722-8133$ & $59.2-90.5$ & [159] \\
\hline Sediment & HPLC-FLD & Dichloromethane & Acetonitrile & Centrifugation & $2.3-6.8 \mathrm{ng} \mathrm{g}^{-1}$ & NA & $>94.0$ & [160] \\
\hline Tap, sea and spring water & GC-FID & Toluene & - & Centrifugation & $20-50$ & $1776-2714$ & $99-103$ & [161] \\
\hline Tap, well, surface water etc. & GC-MS & Chloroform & - & Centrifugation & $1-36$ & NA & - & [162] \\
\hline Tap, spring, surface water etc. & GC-MS & Iso-octane & - & Addition of $\mathrm{NaCl}$ & $0.001-0.009$ & Up to 100000 & - & [163] \\
\hline Tap, rain and wastewater & HPLC-FLD & Cyclohexane & - & Centrifugation & $0.6-62.5$ & $90-247$ & 95-100 & [164] \\
\hline Well, river, lake water etc. & HPLC-FLD & TBAB/2-decanoic acid DES & - & Centrifugation/Solidification & $0.7-6.6$ & $163-198$ & $>80.0$ & [167] \\
\hline Tap, bottle, fountain water etc. & HPLC-FLD & {$\left[\mathrm{C}_{8} \mathrm{MiM}\right]\left[\mathrm{PF}_{6}\right]$} & Acetone & Centrifugation & $0.03-2$ & $301-346$ & - & [169] \\
\hline Tap, well, surface water etc. & HPLC-UV & {$[\mathrm{BBIM}]\left[\mathrm{Tf}_{2} \mathrm{~N}\right]$} & Acetone & Centrifugation & 2 & $2768-5409$ & - & [170] \\
\hline Tap, rain and surface water & HPLC-FLD & Trichloroethylene & Acetonitrile & - & $20-600$ & $86-95$ & - & [172] \\
\hline
\end{tabular}




\subsection{Fabric Phase Sorptive Extraction of PAHs from Environmental Matrices.}

Fabric phase sorptive extraction (FPSE) is a novel sample preparation technique proposed by Kabir and Furton in 2014. FPSE utilizes a natural or synthetic fabric substrate, which is chemically coated in the form of ultra-thin coating with sol-gel organic-inorganic hybrid sorbent as the extraction medium. For the FPSE procedure, the sol-gel sorbent coated FPSE media is immersed into a mixture of appropriate solvents, to remove any undesirable impurities from the material, and rinsed with deionized water to remove residual organic solvents. Subsequently, the FPSE media is submerged into the sample solution placed in a glass vial. A magnet is added into the sample solution, and the sample is magnetically stirred for certain time span for the adsorption of the target analytes. Finally, the FPSE media is removed, and elution of the analytes takes place into another vial containing appropriate elution solvent. Analysis of the eluent can take place after centrifugation and or/filtration [177-180].

FPSE provides high primary contact surface area, thus, rapid and efficient analyte extraction can be easily achieved. Moreover, FPSE is also characterized by low organic solvent consumption, ease in operation, reusability and good selectivity towards the target analytes, which is based directly on the different nature of the fabric substrates and the sol-gel coating. Until today, the use of various fabric substrates, including cellulose, fiber glass and polyester, as well as several sol-gel coatings, including polyethylene glycol, polytetrahydrofuran and polydimethyldiphenylsiloxane have been evaluated as adsorbents for a wide variety of analytes $[17,178,181,182]$.

A trace-level determination of selected PAHs in environmental water samples using FPSE prior to their determination by HPLC-FLD has been reported. For this purpose, a non-polar sol-gel $\mathrm{C}_{18}$ coated FPSE media was prepared and conditioned in a mixture of methanol and acetonitrile for $5 \mathrm{~min}$, and then rinsed with deionized water. The extraction of PAHs took place in a glass vial containing $10 \mathrm{~mL}$ of the aqueous sample solution, in which the sol-gel $C_{18}$ coated FPSE media was directly immersed. After 30 min under constant stirring at $1000 \mathrm{rpm}$, the FPSE media was removed and PAHs were eluted with acetonitrile under ultrasonic radiation for $5 \mathrm{~min}$. The developed FPSE-HPLC-FLD protocol was proved to simple, efficient, fast, sensitive, green, economical and reliable for trace level determination of environmentally important PAHs [183].

Recently, fabric-phase sorptive extraction was coupled with ion mobility spectrometry (IMS) for on-site rapid detection of PAHs in aquatic environment [184]. Ion mobility spectrometry is a rapid and sensitive gas-phase analytical technique, which can be employed for the in the field testing of various chemical compounds, due to its fast analysis and compact size [185]. For the fabrication of the FPSE media, PDMS was coated on the glass fiber cloth through a sol-gel reaction. Glass wool was chosen based on the inlet temperature of IMS, since the thermal desorption of the PAHs was performed after inserting the FPSE media the inlet port of the IMS instrument directly after analyte extraction. Under optimum conditions, low LODs and satisfactory recoveries were obtaining, thus enabling the on-site monitoring water quality.

\subsection{Other Extraction Techniques for the Determination of PAHs in Environmental Matrices.}

Yang et al. [186] synthesized MOF-5 from terephthalic acid and zinc acetate, and evaluated it as a sorbent for the SPE of PAHs from environmental samples. Therefore, $300 \mathrm{mg}$ of MOF- 5 was packed into SPE cartridges. Although MOF-5 is known to be unstable when exposed to water, the researchers reported that the derived material of MOF- 5 still demonstrated good extraction characteristics. This was attributed to the $\pi-\pi$ conjugate effect between the terephthalic acid molecules of the framework and the PAHs and the $\pi$-complexation between PAHs and the central zinc ions. The analytes were further separated and detected in a HPLC-UV system. The method exhibited satisfactory extraction ability and low LODs (0.4-4.0 ng L $\left.{ }^{-1}\right)$, however, no sorbent reusability was reported.

Hu et al. [187] evaluated two zeolitic imidazolate frameworks for the SPE disk extraction of PAHs in aid of filter membrane of PAHs from environmental water samples. The studied ZIFs were both composed of the same metal ion (zinc) and organic linker (benzimidazole), thus differing in spatial structures with one in cube (ZIF-7), while the other was in rhombic dodecahedron (ZIF-11). ZIF-11 
with markedly better extraction efficiencies due to its unique spatial structure with large cages and its molecular composition that was composed of abundant benzyl groups and metal sites on the surface.

ZIF-8 [188] has been also evaluated for the porous membrane-protected micro-solid-phase extraction ( $\mu$-SPE) of PAHs. For this purpose, the sorbent was packed in a sealed porous polypropylene membrane envelope. The novel extraction devices exhibited good extraction characteristics and decreased consumption of the organic solvent.

A molecularly imprinted polymer has been also applied for the SPE of 16 PAHs from seawater, prior to their determination by GC-MS [189]. MIPs were prepared by using the 16 PAHs mixture through non-covalent polymerization as a template based on sol-gel surface imprinting. The developed sorbent exhibited good affinity towards the target analytes. Other examples of novel SPE sorbents that have been implemented for the extraction and preconcentration of PAHs from environmental samples include a cyclodextrin-silica microporous composite [190,191], aminopropyl imidazole-modified silica gel [192], tetraazacalix[2] arene[2]triazine [193], titanium oxide nanotubes [194] and a titanate nanotube array modified by cetyltrimethylammonium bromide [195].

Krupadam et al. [196] prepared MIP microspheres in a continuous segmented flow microfluidic reactor, and used them as packing material for microtraps for the selective extraction of benzo[a]pyrene from environmental water samples. For this purpose, the pumping of monodisperse droplets of acetonitrile containing methacrylic acid as the functional monomer took place, benzo[a]pyrene was used as a template, and ethylene glycol dimethacrylate as cross-linking monomer into the microchannels of the microfluidic reactor. The obtained microspheres exhibited high extraction efficiency and selectivity towards benzo[a]pyrene. In comparison with commercially available activated carbon, the novel microspheres showed 300\% higher adsorption capacity.

A portable system for the in situ extraction of PAHs was proposed by Foan et al. [197]. The researchers designed a microfluidic device for the fast extraction of PAHs using low volume samples. The work was performed on a lab-on-a-chip, made of a silicon/glass microfluidic device functionalized with PDMS. Among the benefits of the novel technique was the low organic solvent consumption and the portability. A comparison of the novel device with SBSE showed approximately 50 times less sample preparation time for the high molecular weight PAHs. However, for the lightest PAHs, the performance of the microchip required improvement.

Flow injection solid-phase extraction (FI-SPE) of PAHs from environmental samples with novel extraction sorbents has been also proposed by the research groups of $\mathrm{Wu}$ [198] and Zhou [199]. The first group used a multi-walled carbon nanotubes (MWCNTs)-packed micro-column for the extraction of PAHs, prior to their determination by GC-MS, while the second group prepared a copper(II) isonicotinate coordination polymer packed in a pre-column for the extraction of PAHs, prior to their determination by HPLC-DAD. Both methods exhibited good extraction characteristics. In the case of GC-MS detection, after the FI-SPE process, the eluates were collected, and manual injection was performed, while for the HPLC-DAD analysis elution of the adsorbed analytes was also performed on-line in the backflush mode by the HPLC mobile phase directly into the chromatographic column, thus minimizing the required analysis steps.

In-syringe solid-phase extraction of PAHs has been also proposed for the on-site sampling of water samples. In-syringe SPE is characterized by portability, simplicity in use, low cost and short extraction time. Zhang et al. [200] evaluated the application of MIL-101 as a novel sorbent, due to its good thermal and mechanical stability, as well as its resistance towards organic solvent and waters. The proposed technique exhibited excellent adsorption performance, since the analytes could be completely adsorbed during one adsorption cycle, thus reducing the extraction time. Moreover, it was found that the adsorbed analytes remained stable on the in-syringe device for at least 7 days.

\section{Conclusions}

A wide variety of novel microextraction techniques and miniaturized extraction techniques have been developed and applied for the extraction of PAHs from environmental samples. Most of the novel 
extraction techniques are variations of the conventional SPE approach, however LLE based extraction approaches have been also developed.

Unequivocally, a lot of progress has been made in the field of sorbent development for micro and miniaturized solid-phase extraction sample preparation techniques. Metal-organic frameworks, covalent organic frameworks, zeolitic imidazole frameworks, graphene, graphene oxide and carbon nanotubes are some typical examples of novel extraction sorbents that have been implemented as such, or after surface modification for the extraction of PAHs from environmental samples. At the same time, ionic liquids, polymeric ionic liquids and deep eutectic solvents have been implemented in liquid phase microextraction approaches as extraction solvents and in solid-phase extraction approaches for the surface modification of a plethora of adsorbents.

The selection of the extraction technique, as well as the extraction sorbent can be based on the needs of the analysis (e.g., selectivity) and the laboratory equipment. MSPE and d-SPE are some examples of simple, rapid and environmentally friendly extraction procedures, that have recently gained a lot of attention, due to their convenience in sample handling. The application of these techniques has rapidly increased, and a wide variety of sorbents have been evaluated. Other novel miniaturized extraction forms, including SBSE, PT-SPE and FPSE have also become popular during the last years. These techniques have been evaluated in less extend compared to SPE, MSPE and d-SPE methods, however, due to their ease in operation, they are considered useful alternatives that enrich the toolbox of analytical chemists.

Proper attention should be also given to the stability of the sorbent in the case of solid-phase extraction, since physical and chemical stability in aqueous solutions is important to provide sorbent reusability, which significantly reduces the cost of the analysis. An important technical problem that has to be overcome is the limited stability of some SPE sorbents (e.g., MOFs) in an aqueous environment that limits their applications, their potential regeneration and recyclability. Another bottleneck of many sample preparation techniques is the lack of compatibility with desorption chambers for HPLC and GC applications.

Therefore, when designing novel sorbents for the extraction of PAHs from environmental matrices, their stability should be carefully examined. Moreover, it is generally preferred to prepare sorbents rich in $\pi$-electron moieties. In this case, the extraction of PAHs can be assisted through $\pi$-stacking, as well as hydrophobic and hydrogen interactions. Additionally, developing materials with an improved affinity towards PAHs, such as MIPs, has also proved to enhance the selectivity and sensitivity of PAHs extraction. Finally, sorbent functionalization with appropriate materials, such as polymers, ionic liquids etc., can also be beneficial for the overall extraction process.

Coupling and combination of different extraction approaches has been also reported for the efficient extraction and enhancement of PAHs from environmental matrices. For this purpose, a solid-phase extraction technique (e.g., SBSE, d-SPE) can be followed by an LPME technique, to further increase the extraction sensitivity.

Future perspectives in the field of sample preparation of environmental samples for PAHs extraction, should focus on developing robust extraction approaches of the on-site extraction and the determination of the environmental contaminants. For this kind of approach, several sorbents and extraction techniques can be examined in order to develop accurate, sensitive and selective sample preparation methods.

Author Contributions: Conceptualization, N.M. and G.A.Z.; writing-original draft preparation, N.M. and G.A.Z.; writing-review and editing, N.M. and G.A.Z.; supervision, G.A.Z. All authors have read and agreed to the published version of the manuscript.

Funding: The research work was supported by the Hellenic Foundation for Research and Innovation (HFRI) under the HFRI PhD Fellowship grant (Fellowship Number: 138).

Conflicts of Interest: The authors declare no conflict of interest. 


\section{References}

1. Cai, Y.; Yan, Z.H.; Wang, N.Y.; Cai, Q.Y.; Yao, S.Z. Preparation of naphthyl functionalized magnetic nanoparticles for extraction of polycyclic aromatic hydrocarbons from river waters. RSC Adv. 2015, 5, 56189-56197. [CrossRef]

2. Pérez, R.A.; Albero, B.; Tadeo, J.L.; Fraile, M.V.; Sánchez-Brunete, C. Determination of PAHs in soil leachates by magnetic solid-phase extraction using nanoparticles and gas chromatography-tandem mass spectrometry. Anal. Methods 2014, 6, 1941-1950. [CrossRef]

3. Zhang, X.; Xie, S.; Paau, M.C.; Zheng, B.; Yuan, H.; Xiao, D.; Choi, M.M.F. Ultrahigh performance liquid chromatographic analysis and magnetic preconcentration of polycyclic aromatic hydrocarbons by Fe3O4-doped polymeric nanoparticles. J. Chromatogr. A 2012, 1247, 1-9. [CrossRef] [PubMed]

4. Boffetta, P.; Jourenkova, N.; Gustavsson, P. Cancer risk from occupational and environmental exposure to polycyclic aromatic hydrocarbons. Cancer Causes Control 1997, 8, 444-472. [CrossRef]

5. Bai, L.; Mei, B.; Guo, Q.Z.; Shi, Z.G.; Feng, Y.Q. Magnetic solid-phase extraction of hydrophobic analytes in environmental samples by a surface hydrophilic carbon-ferromagnetic nanocomposite. J. Chromatogr. A 2010, 1217, 7331-7336. [CrossRef]

6. Han, Q.; Wang, Z.; Xia, J.; Chen, S.; Zhang, X.; Ding, M. Facile and tunable fabrication of $\mathrm{Fe}_{3} \mathrm{O}_{4} / \mathrm{graphene}$ oxide nanocomposites and their application in the magnetic solid-phase extraction of polycyclic aromatic hydrocarbons from environmental water samples. Talanta 2012, 101, 388-395. [CrossRef]

7. Sarafraz-Yazdi, A.; Rokhian, T.; Amiri, A.; Ghaemi, F. Carbon nanofibers decorated with magnetic nanoparticles as a new sorbent for the magnetic solid phase extraction of selected polycyclic aromatic hydrocarbons from water samples. New J. Chem. 2015, 39, 5621-5627. [CrossRef]

8. Azizi, A.; Shahhoseini, F.; Bottaro, C.S. Magnetic molecularly imprinted polymers prepared by reversible addition fragmentation chain transfer polymerization for dispersive solid phase extraction of polycyclic aromatic hydrocarbons in water. J. Chromatogr. A 2020, 1610, 460534. [CrossRef]

9. Manousi, N.; Raber, G.; Papadoyannis, I. Recent Advances in Microextraction Techniques of Antipsychotics in Biological Fluids Prior to Liquid Chromatography Analysis. Separations 2017, 4, 18. [CrossRef]

10. Filippou, O.; Bitas, D.; Samanidou, V. Green approaches in sample preparation of bioanalytical samples prior to chromatographic analysis. J. Chromatogr. B Anal. Technol. Biomed. Life Sci. 2017, 1043, 44-62. [CrossRef]

11. Arthur, C.L.; Pawliszyn, J. Solid Phase Microextraction with Thermal Desorption Using Fused Silica Optical Fibers. Anal. Chem. 1990, 62, 2145-2148. [CrossRef]

12. Liu, H.; Dasgupta, P.K. Analytical chemistry in a drop. solvent extraction in a microdrop. Anal. Chem. 1996, 68, 1817-1821. [CrossRef] [PubMed]

13. Armenta, S.; Garrigues, S.; de la Guardia, M. The role of green extraction techniques in Green Analytical Chemistry. TrAC - Trends Anal. Chem. 2015, 71, 2-8. [CrossRef]

14. Manousi, N.; Gomez-Gomez, B.; Madrid, Y.; Deliyanni, E.A.; Zachariadis, G.A. Determination of rare earth elements by inductively coupled plasma-mass spectrometry after dispersive solid phase extraction with novel oxidized graphene oxide and optimization with response surface methodology and central composite design. Microchem. J. 2020, 152. [CrossRef]

15. Filippou, O.; Deliyanni, E.A.; Samanidou, V.F. Fabrication and evaluation of magnetic activated carbon as adsorbent for ultrasonic assisted magnetic solid phase dispersive extraction of bisphenol A from milk prior to high performance liquid chromatographic analysis with ultraviolet detection. J. Chromatogr. A 2017, 1479, 20-31. [CrossRef]

16. Chen, Z.; Yu, C.; Xi, J.; Tang, S.; Bao, T.; Zhang, J. A hybrid material prepared by controlled growth of a covalent organic framework on amino-modified MIL-68 for pipette tip solid-phase extraction of sulfonamides prior to their determination by HPLC. Microchim. Acta 2019, 186, 393. [CrossRef]

17. Karageorgou, E.; Manousi, N.; Samanidou, V.; Kabir, A.; Furton, K.G. Fabric phase sorptive extraction for the fast isolation of sulfonamides residues from raw milk followed by high performance liquid chromatography with ultraviolet detection. Food Chem. 2016, 196, 428-436. [CrossRef]

18. Nazyropoulou, C.; Samanidou, V. Stir bar sorptive extraction applied to the analysis of biological fluids. Bioanalysis 2015, 7, 2241-2250. [CrossRef] 
19. Li, N.; Jiang, H.L.; Wang, X.; Wang, X.; Xu, G.; Zhang, B.; Wang, L.; Zhao, R.S.; Lin, J.M. Recent advances in graphene-based magnetic composites for magnetic solid-phase extraction. TrAC - Trends Anal. Chem. 2018, 102, 60-74. [CrossRef]

20. Riahi-Zanjani, B.; Balali-Mood, M.; Asoodeh, A.; Es'haghi, Z.; Ghorani-Azam, A. Developing a new sensitive solid-phase microextraction fiber based on carbon nanotubes for preconcentration of morphine. Appl. Nanosci. 2018, 8, 2047-2056. [CrossRef]

21. Manousi, N.; Zachariadis, G.A.; Deliyanni, E.A.; Samanidou, V.F. Applications of metal-organic frameworks in food sample preparation. Molecules 2018, 23, E2896. [CrossRef] [PubMed]

22. Lau, E.V.; Gan, S.; Ng, H.K. Extraction Techniques for Polycyclic Aromatic Hydrocarbons in Soils. Int. J. Anal. Chem. 2010, 2010, 398381. [CrossRef] [PubMed]

23. Pandey, S.K.; Kim, K.H.; Brown, R.J.C. A review of techniques for the determination of polycyclic aromatic hydrocarbons in air. TrAC - Trends Anal. Chem. 2011, 30, 1716-1739. [CrossRef]

24. Plaza-Bolaños, P.; Frenich, A.G.; Vidal, J.L.M. Polycyclic aromatic hydrocarbons in food and beverages. Analytical methods and trends. J. Chromatogr. A 2010, 1217, 6303-6326. [CrossRef]

25. Moret, S.; Conte, L.S. Polycyclic aromatic hydrocarbons in edible fats and oils: Occurrence and analytical methods. J. Chromatogr. A 2000, 882, 245-253. [CrossRef]

26. Locatelli, M.; Forcucci, L.; Sciascia, F.; Cifelli, R.; Ferrone, V.; Carlucci, G. Extraction and Detection Techniques for PAHs Determination in Beverages: A Review. Curr. Chromatogr. 2014, 1, 122-138. [CrossRef]

27. Poster, D.L.; Schantz, M.M.; Sander, L.C.; Wise, S.A. Analysis of polycyclic aromatic hydrocarbons (PAHs) in environmental samples: A critical review of gas chromatographic (GC) methods. Anal. Bioanal. Chem. 2006, 386, 859-881. [CrossRef]

28. Mahgoub, H.A. Extraction Techniques for Determination of Polycyclic Aromatic Hydrocarbons in Water Samples. Int. J. Sci. Res. 2016, 5, NOV152648.

29. Ncube, S.; Madikizela, L.; Cukrowska, E.; Chimuka, L. Recent advances in the adsorbents for isolation of polycyclic aromatic hydrocarbons (PAHs) from environmental sample solutions. TrAC - Trends Anal. Chem. 2018, 99, 101-116. [CrossRef]

30. Islas, G.; Ibarra, I.S.; Hernandez, P.; Miranda, J.M.; Cepeda, A. Dispersive Solid Phase Extraction for the Analysis of Veterinary Drugs Applied to Food Samples: A Review. Int. J. Anal. Chem. 2017, 2017, 8215271. [CrossRef]

31. Han, L.; Sapozhnikova, Y.; Lehotay, S.J. Streamlined sample cleanup using combined dispersive solid-phase extraction and in-vial filtration for analysis of pesticides and environmental pollutants in shrimp. Anal. Chim. Acta 2014, 827, 40-46. [CrossRef] [PubMed]

32. Anastassiades, M.; Lehotay, S.J.; Stajnbaher, D.; Schenck, F. Fast and Easy Multiresidue Method Employing Acetonitrile. J. AOAC Int. 2003, 86, 412-431. [CrossRef] [PubMed]

33. Cvetkovic, J.S.; Mitic, V.D.; Stankov Jovanovic, V.P.; Dimitrijevic, M.V.; Petrovic, G.M.; Nikolic-Mandic, S.D.; Stojanovic, G.S. Optimization of the QuEChERS extraction procedure for the determination of polycyclic aromatic hydrocarbons in soil by gas chromatography-mass spectrometry. Anal. Methods 2016, 8, 1711-1720. [CrossRef]

34. Yaghi, O.M.; Li, H. Hydrothermal Synthesis of a Metal-Organic Framework Containing Large Rectangular Channels. J. Am. Chem. Soc. 1995, 117, 10401-10402. [CrossRef]

35. Farha, O.K.; Eryazici, I.; Jeong, N.C.; Hauser, B.G.; Wilmer, C.E.; Sarjeant, A.A.; Snurr, R.Q.; Nguyen, S.T.; Yazaydin, A.Ö.; Hupp, J.T. Metal-organic framework materials with ultrahigh surface areas: Is the sky the limit? J. Am. Chem. Soc. 2012, 134, 15016-15021. [CrossRef]

36. Manousi, N.; Giannakoudakis, D.A.; Rosenberg, E.; Zachariadis, G.A. Extraction of metal ions with metal-organic frameworks. Molecules 2019, 24, E4605. [CrossRef]

37. Vardali, S.C.; Manousi, N.; Barczak, M.; Giannakoudakis, D.A. Novel approaches utilizing metal-organic framework composites for the extraction of organic compounds and metal traces from fish and seafood. Molecules 2020, 25, E513. [CrossRef]

38. Giliopoulos, D.; Zamboulis, A.; Giannakoudakis, D.; Bikiaris, D.; Triantafyllidis, K. Polymer/metal organic framework (MOF) nanocomposites for biomedical applications. Molecules 2020, 25, 185. [CrossRef]

39. Giannakoudakis, D.A.; Hu, Y.; Florent, M.; Bandosz, T.J. Smart textiles of MOF/g-C3N4 nanospheres for the rapid detection/detoxification of chemical warfare agents. Nanoscale Horizons 2017, 2, 356-364. [CrossRef] 
40. Li, H.; Li, L.; Lin, R.-B.; Zhou, W.; Zhang, Z.; Xiang, S.; Chen, B. Porous metal-organic frameworks for gas storage and separation: Status and challenges. EnergyChem 2019, 1, 100006. [CrossRef]

41. Yang, D.; Gates, B.C. Catalysis by Metal Organic Frameworks: Perspective and Suggestions for Future Research. ACS Catal. 2019, 9, 1779-1798. [CrossRef]

42. Achmann, S.; Hagen, G.; Kita, J.; Malkowsky, I.M.; Kiener, C.; Moos, R. Metal-Organic frameworks for sensing applications in the gas phase. Sensors 2009, 9, 1574-1589. [CrossRef] [PubMed]

43. Rojas, S.; Baati, T.; Njim, L.; Manchego, L.; Neffati, F.; Abdeljelil, N.; Saguem, S.; Serre, C.; Najjar, M.F.; Zakhama, A.; et al. Metal-Organic Frameworks as Efficient Oral Detoxifying Agents. J. Am. Chem. Soc. 2018, 140, 9581-9586. [CrossRef] [PubMed]

44. Wang, L.; Zheng, M.; Xie, Z. Nanoscale metal-organic frameworks for drug delivery: A conventional platform with new promise. J. Mater. Chem. B 2018, 6, 707-717. [CrossRef]

45. Chen, B.; Liang, C.; Yang, J.; Contreras, D.S.; Clancy, Y.L.; Lobkovsky, E.B.; Yaghi, O.M.; Dai, S. A microporous metal-organic framework for gas-chromatographic separation of alkanes. Angew. Chemie - Int. Ed. 2006, 9, 1418-1421. [CrossRef]

46. Gu, Z.Y.; Jiang, J.Q.; Yan, X.P. Fabrication of isoreticular metal-organic framework coated capillary columns for high-resolution gas chromatographic separation of persistent organic pollutants. Anal. Chem. 2011, 83, 5093-5100. [CrossRef]

47. Yang, C.X.; Yan, X.P. Metal-organic framework MIL-101(Cr) for high-performance liquid chromatographic separation of substituted aromatics. Anal. Chem. 2011, 83, 7144-7150. [CrossRef]

48. Yang, C.X.; Chen, Y.J.; Wang, H.F.; Yan, X.P. High-performance separation of fullerenes on metal-organic framework MIL-101(Cr). Chem. - A Eur. J. 2011, 42, 11734-11737. [CrossRef]

49. Xia, L.; Liu, L.; Dou, Y.; Guo, L.; Li, G.; Sun, Z.; You, J. A stable mesoporous metal-organic framework as highly efficient sorbent of dispersive micro solid-phase extraction for the determination of polycyclic aromatic hydrocarbons by HPLC. J. Sep. Sci. 2018, 41, 4331-4339. [CrossRef]

50. Amiri, A.; Ghaemi, F.; Maleki, B. Hybrid nanocomposites prepared from a metal-organic framework of type MOF-199(Cu) and graphene or fullerene as sorbents for dispersive solid phase extraction of polycyclic aromatic hydrocarbons. Microchim. Acta 2019, 186, 1-8. [CrossRef]

51. Chen, B.; Yang, Z.; Zhu, Y.; Xia, Y. Zeolitic imidazolate framework materials: Recent progress in synthesis and applications. J. Mater. Chem. A 2014, 2, 16811-16831. [CrossRef]

52. Liang, X.; Liu, S.; Zhu, R.; Xiao, L.; Yao, S. Highly sensitive analysis of polycyclic aromatic hydrocarbons in environmental water with porous cellulose/zeolitic imidazolate framework-8 composite microspheres as a novel adsorbent coupled with high-performance liquid chromatography. J. Sep. Sci. 2016, 39, 2806-2814. [CrossRef] [PubMed]

53. Mateos, R.; Vera-López, S.; Saz, M.; Díez-Pascual, A.M.; San Andrés, M.P. Graphene/sepiolite mixtures as dispersive solid-phase extraction sorbents for the anaysis of polycyclic aromatic hydrocarbons in wastewater using surfactant aqueous solutions for desorption. J. Chromatogr. A 2019, 1596, 30-40. [CrossRef] [PubMed]

54. Yang, X.P.; Luo, N.; Zong, Y.Y.; Jia, Z.H.; Liao, X.J. Quantum dots extraction coupled with high-performance liquid chromatography for the determination of polycyclic aromatic hydrocarbons in water. Appl. Ecol. Environ. Res. 2017, 15, 171-186.

55. Hemmati, M.; Rajabi, M.; Asghari, A. Magnetic nanoparticle based solid-phase extraction of heavy metal ions: A review on recent advances. Microchim. Acta 2018, 185, 160. [CrossRef]

56. Giakisikli, G.; Anthemidis, A.N. Magnetic materials as sorbents for metal/metalloid preconcentration and/or separation. A review. Anal. Chim. Acta 2013, 789, 1-16. [CrossRef]

57. Manousi, N.; Rosenberg, E.; Deliyanni, E.; Zachariadis, G.A.; Samanidou, V. Magnetic Solid-Phase Extraction of Organic Compounds Based on Graphene Oxide Nanocomposites. Molecules 2020, 25, 1148. [CrossRef]

58. Hesampour, M.; Ali Taher, M.; Behzadi, M. Synthesis, characterization and application of a MnFe2O4@poly(:O-toluidine) nanocomposite for magnetic solid-phase extraction of polycyclic aromatic hydrocarbons. New J. Chem. 2017, 41, 12910-12919. [CrossRef]

59. Rocío-Bautista, P.; Pino, V.; Ayala, J.H.; Pasán, J.; Ruiz-Pérez, C.; Afonso, A.M. A magnetic-based dispersive micro-solid-phase extraction method using the metal-organic framework HKUST-1 and ultra-high-performance liquid chromatography with fluorescence detection for determining polycyclic aromatic hydrocarbons in waters and fruit tea. J. Chromatogr. A 2016, 1426, 42-50. [CrossRef] 
60. Huo, S.H.; Yan, X.P. Facile magnetization of metal-organic framework MIL-101 for magnetic solid-phase extraction of polycyclic aromatic hydrocarbons in environmental water samples. Analyst 2012, 137, 3445-3451. [CrossRef]

61. Zhou, Q.; Lei, M.; Wu, Y.; Yuan, Y. Magnetic solid phase extraction of typical polycyclic aromatic hydrocarbons from environmental water samples with metal organic framework MIL-101 (Cr) modified zero valent iron nano-particles. J. Chromatogr. A 2017, 1487, 22-29. [CrossRef] [PubMed]

62. Du, F.; Qin, Q.; Deng, J.; Ruan, G.; Yang, X.; Li, L.; Li, J. Magnetic metal-organic framework MIL-100(Fe) microspheres for the magnetic solid-phase extraction of trace polycyclic aromatic hydrocarbons from water samples. J. Sep. Sci. 2016, 12, 2356-2364. [CrossRef] [PubMed]

63. Huo, S.H.; An, H.Y.; Yu, J.; Mao, X.F.; Zhang, Z.; Bai, L.; Huang, Y.F.; Zhou, P.X. Pyrolytic in situ magnetization of metal-organic framework MIL-100 for magnetic solid-phase extraction. J. Chromatogr. A 2017, 1517, $18-25$. [CrossRef] [PubMed]

64. Zhang, S.; Yao, W.; Ying, J.; Zhao, H. Polydopamine-reinforced magnetization of zeolitic imidazolate framework ZIF-7 for magnetic solid-phase extraction of polycyclic aromatic hydrocarbons from the air-water environment. J. Chromatogr. A 2016, 1452, 18-26. [CrossRef]

65. He, S.; Zeng, T.; Wang, S.; Niu, H.; Cai, Y. Facile synthesis of magnetic covalent organic framework with three-dimensional bouquet-like structure for enhanced extraction of organic targets. ACS Appl. Mater. Interfaces 2017, 9, 2959-2965. [CrossRef]

66. Wang, R.; Chen, Z. A covalent organic framework-based magnetic sorbent for solid phase extraction of polycyclic aromatic hydrocarbons, and its hyphenation to HPLC for quantitation. Microchim. Acta 2017, 184, 3867-3874. [CrossRef]

67. Rezvani-Eivari, M.; Amiri, A.; Baghayeri, M.; Ghaemi, F. Magnetized graphene layers synthesized on the carbon nanofibers as novel adsorbent for the extraction of polycyclic aromatic hydrocarbons from environmental water samples. J. Chromatogr. A 2016, 1465, 1-8. [CrossRef]

68. Zhang, S.; Niu, H.; Hu, Z.; Cai, Y.; Shi, Y. Preparation of carbon coated $\mathrm{Fe}_{3} \mathrm{O}_{4}$ nanoparticles and their application for solid-phase extraction of polycyclic aromatic hydrocarbons from environmental water samples. J. Chromatogr. A 2010, 1217, 4757-4764. [CrossRef]

69. Mehdinia, A.; Khodaee, N.; Jabbari, A. Fabrication of graphene/ $\mathrm{Fe}_{3} \mathrm{O}_{4} @$ polythiophene nanocomposite and its application in the magnetic solid-phase extraction of polycyclic aromatic hydrocarbons from environmental water samples. Anal. Chim. Acta 2015, 868, 1-9. [CrossRef]

70. Amiri, A.; Baghayeri, M.; Sedighi, M. Magnetic solid-phase extraction of polycyclic aromatic hydrocarbons using a graphene oxide $/ \mathrm{Fe}_{3} \mathrm{O}_{4} @$ polystyrene nanocomposite. Microchim. Acta 2018, 185, 393. [CrossRef]

71. Amiri, A.; Baghayeri, M.; Hamidi, E. Poly(pyrrole-: Co -aniline)@graphene oxide $/ \mathrm{Fe}_{3} \mathrm{O}_{4}$ sorbent for the extraction and preconcentration of polycyclic aromatic hydrocarbons from water samples. New J. Chem. 2018, 42, 16744-16751. [CrossRef]

72. Corps Ricardo, A.I.; Guzmán Bernardo, F.J.; Zougagh, M.; Rodríguez Martín-Doimeadios, R.C.; Ríos, Á. Magnetic nanoparticles-carbon nanotubes hybrid composites for selective solid-phase extraction of polycyclic aromatic hydrocarbons and determination by ultra-high performance liquid chromatography. Anal. Bioanal. Chem. 2017, 409, 5125-5132. [CrossRef] [PubMed]

73. Villar-Navarro, M.; Martín-Valero, M.J.; Fernández-Torres, R.M.; Callejón-Mochón, M.; Bello-López, M.Á. Easy, fast and environmental friendly method for the simultaneous extraction of the 16 EPA PAHs using magnetic molecular imprinted polymers (mag-MIPs). J. Chromatogr. B Anal. Technol. Biomed. Life Sci. 2017, 1044-1045, 63-69. [CrossRef] [PubMed]

74. Benedetti, B.; Di Carro, M.; Magi, E. Multivariate optimization of an extraction procedure based on magnetic molecular imprinted polymer for the determination of polycyclic aromatic hydrocarbons in sea water. Microchem. J. 2019, 145, 1199-1206. [CrossRef]

75. Wang, Y.; Wang, S.; Niu, H.; Ma, Y.; Zeng, T.; Cai, Y.; Meng, Z. Preparation of polydopamine coated $\mathrm{Fe}_{3} \mathrm{O}_{4}$ nanoparticles and their application for enrichment of polycyclic aromatic hydrocarbons from environmental water samples. J. Chromatogr. A 2013, 1283, 20-26. [CrossRef]

76. Xu, S.N.; Zhao, Q.; He, H.B.; Yuan, B.F.; Feng, Y.Q.; Yu, Q.W. Rapid determination of polycyclic aromatic hydrocarbons in environmental water based on magnetite nanoparticles/polypyrrole magnetic solid-phase extraction. Anal. Methods 2014, 6, 7046-7053. [CrossRef] 
77. Nurerk, P.; Kanatharana, P.; Bunkoed, O. Polyaniline-coated magnetite nanoparticles incorporated in alginate beads for the extraction and enrichment of polycyclic aromatic hydrocarbons in water samples. Int. J. Environ. Anal. Chem. 2017, 2, 145-158. [CrossRef]

78. Galán-Cano, F.; Del Carmen Alcudia-León, M.; Lucena, R.; Cárdenas, S.; Valcárcel, M. Ionic liquid coated magnetic nanoparticles for the gas chromatography/mass spectrometric determination of polycyclic aromatic hydrocarbons in waters. J. Chromatogr. A 2013, 1300, 134-140. [CrossRef]

79. Bakhshaei, S.; Kamboh, M.A.; Nodeh, H.R.; Md Zain, S.; Mahmad Rozi, S.K.; Mohamad, S.; Mohammed Mohialdeen, I.A. Magnetic solid phase extraction of polycyclic aromatic hydrocarbons and chlorophenols based on cyano-ionic liquid functionalized magnetic nanoparticles and their determination by HPLC-DAD. RSC Adv. 2016, 6, 77047-77058. [CrossRef]

80. Shahriman, M.S.; Ramachandran, M.R.; Zain, N.N.M.; Mohamad, S.; Manan, N.S.A.; Yaman, S.M. Polyaniline-dicationic ionic liquid coated with magnetic nanoparticles composite for magnetic solid phase extraction of polycyclic aromatic hydrocarbons in environmental samples. Talanta 2018, 178, 211-221. [CrossRef] [PubMed]

81. Liu, X.; Lu, X.; Huang, Y.; Liu, C.; Zhao, S. $\mathrm{Fe}_{3} \mathrm{O}_{4} @$ ionic liquid@methyl orange nanoparticles as a novel nano-adsorbent for magnetic solid-phase extraction of polycyclic aromatic hydrocarbons in environmental water samples. Talanta 2014, 119, 341-347. [CrossRef] [PubMed]

82. Zhang, S.; Niu, H.; Zhang, Y.; Liu, J.; Shi, Y.; Zhang, X.; Cai, Y. Biocompatible phosphatidylcholine bilayer coated on magnetic nanoparticles and their application in the extraction of several polycyclic aromatic hydrocarbons from environmental water and milk samples. J. Chromatogr. A 2012, 1238, 38-45. [CrossRef] [PubMed]

83. Xue, S.W.; Tang, M.Q.; Xu, L.; Shi, Z. guo Magnetic nanoparticles with hydrophobicity and hydrophilicity for solid-phase extraction of polycyclic aromatic hydrocarbons from environmental water samples. J. Chromatogr. A 2015, 1411, 9-16. [CrossRef] [PubMed]

84. Long, Y.; Chen, Y.; Yang, F.; Chen, C.; Pan, D.; Cai, Q.; Yao, S. Triphenylamine-functionalized magnetic microparticles as a new adsorbent coupled with high performance liquid chromatography for the analysis of trace polycyclic aromatic hydrocarbons in aqueous samples. Analyst 2012, 137, 2716-2722. [CrossRef] [PubMed]

85. Liu, Y.; Li, H.; Lin, J.M. Magnetic solid-phase extraction based on octadecyl functionalization of monodisperse magnetic ferrite microspheres for the determination of polycyclic aromatic hydrocarbons in aqueous samples coupled with gas chromatography-mass spectrometry. Talanta 2009, 77, 1037-1042. [CrossRef]

86. Ballesteros-Gómez, A.; Rubio, S. Hemimicelles of alkyl carboxylates chemisorbed onto magnetic nanoparticles: Study and application to the extraction of carcinogenic polycyclic aromatic hydrocarbons in environmental water samples. Anal. Chem. 2009, 81, 9012-9020. [CrossRef]

87. Ding, J.; Gao, Q.; Luo, D.; Shi, Z.G.; Feng, Y.Q. n-Octadecylphosphonic acid grafted mesoporous magnetic nanoparticle: Preparation, characterization, and application in magnetic solid-phase extraction. J. Chromatogr. A 2010, 1217, 7351-7358. [CrossRef]

88. Reyes-Gallardo, E.M.; Lucena, R.; Cárdenas, S.; Valcárcel, M. Magnetic nanoparticles-nylon 6 composite for the dispersive micro solid phase extraction of selected polycyclic aromatic hydrocarbons from water samples. J. Chromatogr. A 2014, 1345, 43-49. [CrossRef]

89. Wang, H.; Zhao, X.; Meng, W.; Wang, P.; Wu, F.; Tang, Z.; Han, X.; Giesy, J.P. Cetyltrimethylammonium Bromide-Coated $\mathrm{Fe}_{3} \mathrm{O}_{4}$ Magnetic Nanoparticles for Analysis of 15 Trace Polycyclic Aromatic Hydrocarbons in Aquatic Environments by Ultraperformance, Liquid Chromatography With Fluorescence Detection. Anal. Chem. 2015, 87, 7667-7675. [CrossRef]

90. Rozi, S.K.M.; Nodeh, H.R.; Kamboh, M.A.; Manan, N.S.A.; Mohamad, S. Novel palm fatty acid functionalized magnetite nanoparticles for magnetic solid-phase extraction of trace polycyclic aromatic hydrocarbons from environmental samples. J. Oleo Sci. 2017, 66, 771-784. [CrossRef]

91. Zou, Y.; Chen, Y.; Yan, Z.; Chen, C.; Wang, J.; Yao, S. Magnetic solid-phase extraction based on tetrabenzyl modified $\mathrm{Fe}_{3} \mathrm{O}_{4}$ nanoparticles for the analysis of trace polycyclic aromatic hydrocarbons in environmental water samples. Analyst 2013, 138, 5904-5912. [CrossRef] [PubMed] 
92. Zhang, W.; Zhang, Y.; Jiang, Q.; Zhao, W.; Yu, A.; Chang, H.; Lu, X.; Xie, F.; Ye, B.; Zhang, S. Tetraazacalix[2]arence[2] triazine Coated $\mathrm{Fe}_{3} \mathrm{O}_{4} / \mathrm{SiO}_{2}$ Magnetic Nanoparticles for Simultaneous Dispersive Solid Phase Extraction and Determination of Trace Multitarget Analytes. Anal. Chem. 2016, 88, 10523-10532. [CrossRef]

93. Al-rashdi, A.A. Double-functionalized magnetic nanoparticles for preconcentration and determination of polycyclic aromatic hydrocarbons in water samples. Anal. Chem. Res. 2016, 10,9-17. [CrossRef]

94. Feng, X.; Ding, X.; Jiang, D. Covalent organic frameworks. Chem. Soc. Rev. 2012, 41, 6010-6022. [CrossRef] [PubMed]

95. Li, N.; Du, J.; Wu, D.; Liu, J.; Li, N.; Sun, Z.; Li, G.; Wu, Y. Recent advances in facile synthesis and applications of covalent organic framework materials as superior adsorbents in sample pretreatment. TrAC - Trends Anal. Chem. 2018, 108, 154-166. [CrossRef]

96. Novoselov, K.S.; Geim, A.K.; Morozov, S.V.; Jiang, D.; Zhang, Y.; Dubonos, S.V.; Grigorieva, I.V.; Firsov, A.A. Electric field in atomically thin carbon films. Science 2004, 306, 666-669. [CrossRef]

97. González-Fuenzalida, R.A.; López-García, E.; Moliner-Martínez, Y.; Campíns-Falcó, P. Adsorbent phases with nanomaterials for in-tube solid-phase microextraction coupled on-line to liquid nanochromatography. J. Chromatogr. A 2016, 1432, 17-25. [CrossRef]

98. Bitas, D.; Samanidou, V. Molecularly imprinted polymers as extracting media for the chromatographic determination of antibiotics in milk. Molecules 2018, 32, E316. [CrossRef]

99. Queiroz, M.E.C.; Melo, L.P. Selective capillary coating materials for in-tube solid-phase microextraction coupled to liquid chromatography to determine drugs and biomarkers in biological samples: A review. Anal. Chim. Acta 2014, 826, 1-11. [CrossRef]

100. Tamayo, F.G.; Turiel, E.; Martín-Esteban, A. Molecularly imprinted polymers for solid-phase extraction and solid-phase microextraction: Recent developments and future trends. J. Chromatogr. A 2007, 1152, 32-40. [CrossRef]

101. Kissoudi, M.; Samanidou, V. Recent advances in applications of ionic liquids in miniaturized microextraction techniques. Molecules 2018, 23, E1437. [CrossRef]

102. Chatzimitakos, T.; Stalikas, C. Carbon-based nanomaterials functionalized with ionic liquids for microextraction in sample preparation. Separations 2017, 4, 14. [CrossRef]

103. Han, D.; Row, K.H. Recent applications of ionic liquids in separation technology. Molecules 2010, 15, 2405-2426. [CrossRef] [PubMed]

104. Kataoka, H.; Lord, H.L.; Pawliszyn, J. Applications of solid-phase microextraction in food analysis. J. Chromatogr. A 2000, 880, 35-62. [CrossRef]

105. Aulakh, J.S.; Malik, A.K.; Kaur, V.; Schmitt-Kopplin, P. A review on solid phase micro extraction - High performance liquid chromatography (SPME-HPLC) analysis of pesticides. Crit. Rev. Anal. Chem. 2005, 35, 71-85. [CrossRef]

106. Chen, C.; Liang, X.; Wang, J.; Yang, S.; Yan, Z.; Cai, Q.; Yao, S. Development of a highly robust solid phase microextraction fiber based on crosslinked methyl methacrylate-polyhedral oligomeric silsesquioxane hybrid polymeric coating. Anal. Chim. Acta 2013, 792, 45-51. [CrossRef]

107. Rocío-Bautista, P.; Pacheco-Fernández, I.; Pasán, J.; Pino, V. Are metal-organic frameworks able to provide a new generation of solid-phase microextraction coatings? - A review. Anal. Chim. Acta 2016, 939, $26-41$. [CrossRef]

108. Yu, H.; Ho, T.D.; Anderson, J.L. Ionic liquid and polymeric ionic liquid coatings in solid-phase microextraction. TrAC - Trends Anal. Chem. 2013, 45, 219-232. [CrossRef]

109. López-Darias, J.; Pino, V.; Anderson, J.L.; Graham, C.M.; Afonso, A.M. Determination of water pollutants by direct-immersion solid-phase microextraction using polymeric ionic liquid coatings. J. Chromatogr. A 2010, 1217, 1236-1243. [CrossRef]

110. Meng, Y.; Anderson, J.L. Tuning the selectivity of polymeric ionic liquid sorbent coatings for the extraction of polycyclic aromatic hydrocarbons using solid-phase microextraction. J. Chromatogr. A 2010, 1217, 6143-6152. [CrossRef]

111. Feng, J.; Sun, M.; Li, J.; Liu, X.; Jiang, S. A novel aromatically functional polymeric ionic liquid as sorbent material for solid-phase microextraction. J. Chromatogr. A 2012, 1227, 54-59. [CrossRef] [PubMed] 
112. López-Darias, J.; Pino, V.; Meng, Y.; Anderson, J.L.; Afonso, A.M. Utilization of a benzyl functionalized polymeric ionic liquid for the sensitive determination of polycyclic aromatic hydrocarbons; parabens and alkylphenols in waters using solid-phase microextraction coupled to gas chromatography-flame ionization detection. J. Chromatogr. A 2010, 1217, 7189-7197. [PubMed]

113. Zhang, S.; Du, Z.; Li, G. Layer-by-layer fabrication of chemical-bonded graphene coating for solid-phase microextraction. Anal. Chem. 2011, 83, 7531-7541. [CrossRef] [PubMed]

114. Wang, F.; Zheng, Y.; Qiu, J.; Liu, S.; Tong, Y.; Zhu, F.; Ouyang, G. Graphene-based metal and nitrogen-doped carbon composites as adsorbents for highly sensitive solid phase microextraction of polycyclic aromatic hydrocarbons. Nanoscale 2018, 10, 10073-10078. [CrossRef]

115. Xu, L.; Feng, J.; Liang, X.; Li, J.; Jiang, S. C 18 functionalized graphene oxide as a novel coating for solid-phase microextraction. J. Sep. Sci. 2012, 35, 1531-1537. [CrossRef]

116. Sun, M.; Feng, J.; Bu, Y.; Duan, H.; Wang, X.; Luo, C. Development of a solid-phase microextraction fiber by the chemical binding of graphene oxide on a silver-coated stainless-steel wire with an ionic liquid as the crosslinking agent. J. Sep. Sci. 2014, 37, 3691-3698. [CrossRef]

117. Xu, L.; Feng, J.; Li, J.; Liu, X.; Jiang, S. Graphene oxide bonded fused-silica fiber for solid-phase microextraction-gas chromatography of polycyclic aromatic hydrocarbons in water. J. Sep. Sci. 2012, 35, 93-100. [CrossRef]

118. Behzadi, M.; Noroozian, E.; Mirzaei, M. A novel coating based on carbon nanotubes/poly-ortho-phenylenediamine composite for headspace solid-phase microextraction of polycyclic aromatic hydrocarbons. Talanta 2013, 108, 66-73. [CrossRef]

119. Maghsoudi, S.; Noroozian, E. HP-SPME of volatile polycyclic aromatic hydrocarbons from water using multiwalled carbon nanotubes coated on a steel fiber through electrophoretic deposition. Chromatographia 2012, 75, 913-921. [CrossRef]

120. Zhang, X.; Zang, X.H.; Wang, J.T.; Wang, C.; Wu, Q.H.; Wang, Z. Porous carbon derived from aluminum-based metal organic framework as a fiber coating for the solid-phase microextraction of polycyclic aromatic hydrocarbons from water and soil. Microchim. Acta 2015, 182, 2353-2359. [CrossRef]

121. Gutiérrez-Serpa, A.; Pacheco-Fernández, I.; Pasán, J.; Pino, V. Metal-organic frameworks as key materials for solid-phase microextraction devices-a review. Separations 2019, 6, 47. [CrossRef]

122. Sun, S.; Huang, L.; Xiao, H.; Shuai, Q.; Hu, S. In situ self-transformation metal into metal-organic framework membrane for solid-phase microextraction of polycyclic aromatic hydrocarbons. Talanta 2019, 202, 145-151. [CrossRef] [PubMed]

123. Zhang, G.; Zang, X.; Li, Z.; Wang, C.; Wang, Z. Polydimethylsiloxane/metal-organic frameworks coated fiber for solid-phase microextraction of polycyclic aromatic hydrocarbons in river and lake water samples. Talanta 2014, 129, 600-605. [CrossRef] [PubMed]

124. Kong, J.; Zhu, F.; Huang, W.; He, H.; Hu, J.; Sun, C.; Xian, Q.; Yang, S. Sol-gel based metal-organic framework zeolite imidazolate framework-8 fibers for solid-phase microextraction of nitro polycyclic aromatic hydrocarbons and polycyclic aromatic hydrocarbons in water samples. J. Chromatogr. A 2019, 1603, 92-101. [CrossRef] [PubMed]

125. Amanzadeh, H.; Yamini, Y.; Masoomi, M.Y.; Morsali, A. Nanostructured metal-organic frameworks, TMU-4, TMU-5, and TMU-6, as novel adsorbents for solid phase microextraction of polycyclic aromatic hydrocarbons. New J. Chem. 2017, 20, 12035-12043. [CrossRef]

126. Wang, G.; Lei, Y.; Song, H. Exploration of metal-organic framework MOF-177 coated fibers for headspace solid-phase microextraction of polychlorinated biphenyls and polycyclic aromatic hydrocarbons. Talanta 2015, 144, 369-374. [CrossRef]

127. Gao, J.; Huang, C.; Lin, Y.; Tong, P.; Zhang, L. In situ solvothermal synthesis of metal-organic framework coated fiber for highly sensitive solid-phase microextraction of polycyclic aromatic hydrocarbons. J. Chromatogr. A 2016, 1436, 1-8. [CrossRef]

128. Li, Q.L.; Wang, X.; Chen, X.F.; Wang, M.L.; Zhao, R.S. In situ hydrothermal growth of ytterbium-based metal-organic framework on stainless steel wire for solid-phase microextraction of polycyclic aromatic hydrocarbons from environmental samples. J. Chromatogr. A 2015, 1415, 11-19. [CrossRef]

129. Huo, S.H.; Yu, J.; Fu, Y.Y.; Zhou, P.X. In situ hydrothermal growth of a dual-ligand metal-organic framework film on a stainless steel fiber for solid-phase microextraction of polycyclic aromatic hydrocarbons in environmental water samples. RSC Adv. 2016, 6, 14042-14048. [CrossRef] 
130. Liu, M.; Liu, J.; Guo, C.; Li, Y. Metal azolate framework-66-coated fiber for headspace solid-phase microextraction of polycyclic aromatic hydrocarbons. J. Chromatogr. A 2019, 1584, 57-63. [CrossRef]

131. Mehdinia, A.; Mohammadi, A.A.; Davarani, S.S.H.; Banitaba, M.H. Application of self-assembled monolayers in the preparation of solid-phase microextraction coatings. Chromatographia 2011, 5-6, 421-427. [CrossRef]

132. Yang, L.; Zhang, J.; Zhao, F.; Zeng, B. Electrodeposition of self-assembled poly(3,4-ethylenedioxythiophene) @gold nanoparticles on stainless steel wires for the headspace solid-phase microextraction and gas chromatographic determination of several polycyclic aromatic hydrocarbons. J. Chromatogr. A 2016, 1471, 80-86. [CrossRef] [PubMed]

133. Harati, F.; Ghiasvand, A.; Dalvand, K.; Haddad, P.R. Fused-silica capillary internally modified with nanostructured octadecyl silica for dynamic in-tube solid-phase microextraction of polycyclic aromatic hydrocarbons from aqueous media. Microchem. J. 2020, 155, 104672. [CrossRef]

134. Ghiasvand, A.; Yazdankhah, F.; Paull, B. Heating-, Cooling- and Vacuum-Assisted Solid-Phase Microextraction (HCV-SPME) for Efficient Sampling of Environmental Pollutants in Complex Matrices. Chromatographia 2020, 83, 531-540. [CrossRef]

135. Kremser, A.; Jochmann, M.A.; Schmidt, T.C. PAL SPME Arrow - Evaluation of a novel solid-phase microextraction device for freely dissolved PAHs in water. Anal. Bioanal. Chem. 2016, 408, 943-952. [CrossRef]

136. Baltussen, E.; Sandra, P.; David, F.; Cramers, C. Stir bar sorptive extraction (SBSE), a novel extraction technique for aqueous samples: Theory and principles. J. Microcolumn Sep. 1999, 10, 737-747. [CrossRef]

137. David, F.; Sandra, P. Stir bar sorptive extraction for trace analysis. J. Chromatogr. A 2007, 1152, 54-69. [CrossRef]

138. García-Falcón, M.S.; Cancho-Grande, B.; Simal-Gándara, J. Stirring bar sorptive extraction in the determination of PAHs in drinking waters. Water Res. 2004, 38, 1679-1684. [CrossRef]

139. Popp, P.; Bauer, C.; Wennrich, L. Application of stir bar sorptive extraction in combination with column liquid chromatography for the determination of polycyclic aromatic hydrocarbons in water samples. Anal. Chim. Acta 2001, 436, 1-9. [CrossRef]

140. Popp, P.; Bauer, C.; Hauser, B.; Keil, P.; Wennrich, L. Extraction of polycyclic aromatic hydrocarbons and organochloride compounds from water: A comparison between solid-phase microextraction and stir bar sorptive extraction. J. Sep. Sci. 2003, 9-10, 961-967. [CrossRef]

141. Bourdat-Deschamps, M.; Daudin, J.J.; Barriuso, E. An experimental design approach to optimise the determination of polycyclic aromatic hydrocarbons from rainfall water using stir bar sorptive extraction and high performance liquid chromatography-fluorescence detection. J. Chromatogr. A 2007, 1167, 143-153. [CrossRef] [PubMed]

142. Mao, X.; Hu, B.; He, M.; Fan, W. Stir bar sorptive extraction approaches with a home-made portable electric stirrer for the analysis of polycyclic aromatic hydrocarbon compounds in environmental water. J. Chromatogr. A 2012, 1260, 16-24. [CrossRef] [PubMed]

143. Mollahosseini, A.; Rokue, M.; Mojtahedi, M.M.; Toghroli, M.; Kamankesh, M.; Motaharian, A. Mechanical stir bar sorptive extraction followed by gas chromatography as a new method for determining polycyclic aromatic hydrocarbons in water samples. Microchem. J. 2016, 126, 431-437. [CrossRef]

144. Huang, X.; Yuan, D. Preparation of stir bars for sorptive extraction based on monolithic material. J. Chromatogr. A 2007, 1154, 152-157. [CrossRef] [PubMed]

145. Ekbatani Amlashi, N.; Hadjmohammadi, M.R. Sol-gel coating of poly(ethylene glycol)-grafted multiwalled carbon nanotubes for stir bar sorptive extraction and its application to the analysis of polycyclic aromatic hydrocarbons in water. J. Sep. Sci. 2016, 39, 3445-3456. [CrossRef] [PubMed]

146. Hu, C.; He, M.; Chen, B.; Zhong, C.; Hu, B. Sorptive extraction using polydimethylsiloxane/metal-organic framework coated stir bars coupled with high performance liquid chromatography-fluorescence detection for the determination of polycyclic aromatic hydrocarbons in environmental water samples. J. Chromatogr. A 2014, 1356, 45-53. [CrossRef]

147. Benedé, J.L.; Anderson, J.L.; Chisvert, A. Trace determination of volatile polycyclic aromatic hydrocarbons in natural waters by magnetic ionic liquid-based stir bar dispersive liquid microextraction. Talanta 2018, 176, 253-261. [CrossRef] 
148. Luo, Y.B.; Cheng, J.S.; Ma, Q.; Feng, Y.Q.; Li, J.H. Graphene-polymer composite: Extraction of polycyclic aromatic hydrocarbons from water samples by stir rod sorptive extraction. Anal. Methods 2011, 3, 92-98. [CrossRef]

149. Luo, Y.B.; Ma, Q.; Feng, Y.Q. Stir rod sorptive extraction with monolithic polymer as coating and its application to the analysis of fluoroquinolones in honey sample. J. Chromatogr. A 2010, 1217, 3583-3589. [CrossRef]

150. Yu, C.; Hu, B. Automated stir plate (bar) sorptive extraction coupled to high-performance liquid chromatography for the determination of polycyclic aromatic hydrocarbons. J. Sep. Sci. 2010, 33, 2176-2183. [CrossRef]

151. Rutkowska, M.; Płotka-Wasylka, J.; Sajid, M.; Andruch, V. Liquid-phase microextraction: A review of reviews. Microchem. J. 2019, 149, 103989. [CrossRef]

152. Bello-López, M.Á.; Ramos-Payán, M.; Ocaña-González, J.A.; Fernández-Torres, R.; Callejón-Mochón, M. Analytical Applications of Hollow Fiber Liquid Phase Microextraction (HF-LPME): A Review. Anal. Lett. 2012, 45, 804-830. [CrossRef]

153. Kokosa, J.M. Recent trends in using single-drop microextraction and related techniques in green analytical methods. TrAC - Trends Anal. Chem. 2015, 71, 194-204. [CrossRef]

154. Kocúrová, L.; Balogh, I.S.; Šandrejová, J.; Andruch, V. Recent advances in dispersive liquid-liquid microextraction using organic solvents lighter than water. A review. Microchem. J. 2012, 102, 11-17. [CrossRef]

155. Rykowska, I.; Ziemblińska, J.; Nowak, I. Modern approaches in dispersive liquid-liquid microextraction (DLLME) based on ionic liquids: A review. J. Mol. Liq. 2018, 259, 319-339. [CrossRef]

156. Rezaee, M.; Assadi, Y.; Milani Hosseini, M.R.; Aghaee, E.; Ahmadi, F.; Berijani, S. Determination of organic compounds in water using dispersive liquid-liquid microextraction. J. Chromatogr. A 2006, 1116, 1-9. [CrossRef]

157. Guo, L.; Lee, H.K. Low-density solvent-based solvent demulsification dispersive liquid-liquid microextraction for the fast determination of trace levels of sixteen priority polycyclic aromatic hydrocarbons in environmental water samples. J. Chromatogr. A 2011, 1218, 5040-5046. [CrossRef]

158. Hosseini, M.H.; Rezaee, M.; Akbarian, S.; Mizani, F.; Pourjavid, M.R.; Arabieh, M. Homogeneous liquid-liquid microextraction via flotation assistance for rapid and efficient determination of polycyclic aromatic hydrocarbons in water samples. Anal. Chim. Acta 2013, 762, 54-60. [CrossRef]

159. Song, X.; Li, J.; Liao, C.; Chen, L. Ultrasound-assisted dispersive liquid-liquid microextraction combined with low solvent consumption for determination of polycyclic aromatic hydrocarbons in seawater by GC-MS. Chromatographia 2011, 74, 89-98. [CrossRef]

160. Leng, G.; Lui, G.; Chen, Y.; Yin, H.; Dan, D. Vortex-assisted extraction combined with dispersive liquid-liquid microextraction for the determination of polycyclic aromatic hydrocarbons in sediment by high performance liquid chromatography. J. Sep. Sci. 2012, 35, 2796-2804. [CrossRef]

161. Saleh, A.; Yamini, Y.; Faraji, M.; Rezaee, M.; Ghambarian, M. Ultrasound-assisted emulsification microextraction method based on applying low density organic solvents followed by gas chromatography analysis for the determination of polycyclic aromatic hydrocarbons in water samples. J. Chromatogr. A 2009, 1216, 6673-6679. [CrossRef] [PubMed]

162. Ozcan, S.; Tor, A.; Aydin, M.E. Determination of polycyclic aromatic hydrocarbons in waters by ultrasound-assisted emulsification-microextraction and gas chromatography-mass spectrometry. Anal. Chim. Acta 2010, 665, 193-199. [CrossRef] [PubMed]

163. Avino, P.; Notardonato, I.; Perugini, L.; Russo, M.V. New protocol based on high-volume sampling followed by DLLME-GC-IT/MS for determining PAHs at ultra-trace levels in surface water samples. Microchem. J. 2017, 133, 251-257. [CrossRef]

164. Cheng, J.; Matsadiq, G.; Liu, L.; Zhou, Y.W.; Chen, G. Development of a novel ultrasound-assisted surfactant-enhanced emulsification microextraction method and its application to the analysis of eleven polycyclic aromatic hydrocarbons at trace levels in water. J. Chromatogr. A 2011, 1218, 2476-2482. [CrossRef] [PubMed]

165. Mansour, F.R.; Danielson, N.D. Solidification of floating organic droplet in dispersive liquid-liquid microextraction as a green analytical tool. Talanta 2017, 170, 22-35. [CrossRef]

166. Guiñez, M.; Martinez, L.D.; Fernandez, L.; Cerutti, S. Dispersive liquid-liquid microextraction based on solidification of floating organic drop and fluorescence detection for the determination of nitrated polycyclic aromatic hydrocarbons in aqueous samples. Microchem. J. 2017, 131, 1-8. [CrossRef] 
167. Yousefi, S.M.; Shemirani, F.; Ghorbanian, S.A. Hydrophobic Deep Eutectic Solvents in Developing Microextraction Methods Based on Solidification of Floating Drop: Application to the Trace HPLC/FLD Determination of PAHs. Chromatographia 2018 81, 1201-1211. [CrossRef]

168. Shishov, A.; Bulatov, A.; Locatelli, M.; Carradori, S.; Andruch, V. Application of deep eutectic solvents in analytical chemistry. A review. Microchem. J. 2017, 135, 33-38. [CrossRef]

169. Pena, M.T.; Casais, M.C.; Mejuto, M.C.; Cela, R. Development of an ionic liquid based dispersive liquid-liquid microextraction method for the analysis of polycyclic aromatic hydrocarbons in water samples. J. Chromatogr. A 2009, 1216, 6356-6364. [CrossRef]

170. Liu, L.; He, L.; Jiang, X.; Zhao, W.; Xiang, G.; Anderson, J.L. Macrocyclic polyamine-functionalized silica as a solid-phase extraction material coupled with ionic liquid dispersive liquid-liquid extraction for the enrichment of polycyclic aromatic hydrocarbons. J. Sep. Sci. 2014, 37, 1004-1011. [CrossRef]

171. Shamsipur, M.; Hashemi, B. Extraction and determination of polycyclic aromatic hydrocarbons in water samples using stir bar sorptive extraction (SBSE) combined with dispersive liquid-liquid microextraction based on the solidification of floating organic drop (DLLME-SFO) followed by. RSC Adv. 2015, 5, 20339-20345. [CrossRef]

172. Fernández, M.; Clavijo, S.; Forteza, R.; Cerdà, V. Determination of polycyclic aromatic hydrocarbons using lab on valve dispersive liquid-liquid microextraction coupled to high performance chromatography. Talanta 2015, 138, 190-195. [CrossRef] [PubMed]

173. Pena-Pereira, F.; Costas-Mora, I.; Lavilla, I.; Bendicho, C. Rapid screening of polycyclic aromatic hydrocarbons (PAHs) in waters by directly suspended droplet microextraction-microvolume fluorospectrometry. Talanta 2012, 89, 217-222. [CrossRef] [PubMed]

174. Santos, L.O.; dos Anjos, J.P.; Ferreira, S.L.C.; de Andrade, J.B. Simultaneous determination of PAHS, nitro-PAHS and quinones in surface and groundwater samples using SDME/GC-MS. Microchem. J. 2017, 133, 431-440. [CrossRef]

175. Sibiya, P.; Cukrowska, E.; Jönsson, J.Å.; Chimuka, L. Hollow-fibre liquid-phase microextraction for the determination of polycyclic aromatic hydrocarbons in Johannesburg Jukskei River, South Africa. Chromatographia 2013, 76, 427-436. [CrossRef]

176. Ratola, N.; Alves, A.; Kalogerakis, N.; Psillakis, E. Hollow-fibre liquid-phase microextraction: A simple and fast cleanup step used for PAHs determination in pine needles. Anal. Chim. Acta 2008, 618, 70-78. [CrossRef]

177. Aznar, M.; Alfaro, P.; Nerin, C.; Kabir, A.; Furton, K.G. Fabric phase sorptive extraction: An innovative sample preparation approach applied to the analysis of specific migration from food packaging. Anal. Chim. Acta 2016, 936, 97-107. [CrossRef]

178. Zilfidou, E.; Kabir, A.; Furton, K.G.; Samanidou, V. Fabric phase sorptive extraction: Current state of the art and future perspectives. Separations 2018, 5, 40. [CrossRef]

179. Kabir, A.; Furton, K.G. Sample preparation in food analysis: Practices, problems and future outlook. In Analytical Chemistry: Developments, Applications and Challenges in Food Analysis; Nova Science Publishers, Inc.: Hauppauge, NY, USA, 2017; ISBN 9781536122824.

180. Locatelli, M.; Kabir, A.; Innosa, D.; Lopatriello, T.; Furton, K.G. A fabric phase sorptive extraction-High performance liquid chromatography-Photo diode array detection method for the determination of twelve azole antimicrobial drug residues in human plasma and urine. J. Chromatogr. B Anal. Technol. Biomed. Life Sci. 2017, 1040, 192-198. [CrossRef]

181. Samanidou, V.; Galanopoulos, L.D.; Kabir, A.; Furton, K.G. Fast extraction of amphenicols residues from raw milk using novel fabric phase sorptive extraction followed by high-performance liquid chromatography-diode array detection. Anal. Chim. Acta 2015, 855, 41-50. [CrossRef]

182. Samanidou, V.; Michaelidou, K.; Kabir, A.; Furton, K.G. Fabric phase sorptive extraction of selected penicillin antibiotic residues from intact milk followed by high performance liquid chromatography with diode array detection. Food Chem. 2017, 224, 131-138. [CrossRef] [PubMed]

183. Saini, S.S.; Kabir, A.; Rao, A.L.J.; Malik, A.K.; Furton, K.G. A novel protocol to monitor trace levels of selected polycyclic aromatic hydrocarbons in environmental water using fabric phase sorptive extraction followed by high performance liquid chromatography-fluorescence detection. Separations 2017, 4, 22. [CrossRef]

184. Sun, T.; Wang, D.; Tang, Y.; Xing, X.; Zhuang, J.; Cheng, J.; Du, Z. Fabric-phase sorptive extraction coupled with ion mobility spectrometry for on-site rapid detection of PAHs in aquatic environment. Talanta 2019, 195, 109-116. [CrossRef] [PubMed] 
185. Armenta, S.; Alcala, M.; Blanco, M. A review of recent, unconventional applications of ion mobility spectrometry (IMS). Anal. Chim. Acta 2011, 703, 114-123. [CrossRef] [PubMed]

186. Yang, S.; Chen, C.; Yan, Z.; Cai, Q.; Yao, S. Evaluation of metal-organic framework 5 as a new SPE material for the determination of polycyclic aromatic hydrocarbons in environmental waters. J. Sep. Sci. 2013, 36, 1283-1290. [CrossRef] [PubMed]

187. Hu, H.; Liu, S.; Chen, C.; Wang, J.; Zou, Y.; Lin, L.; Yao, S. Two novel zeolitic imidazolate frameworks (ZIFs) as sorbents for solid-phase extraction (SPE) of polycyclic aromatic hydrocarbons (PAHs) in environmental water samples. Analyst 2014, 139, 5818-5826. [CrossRef]

188. Ge, D.; Lee, H.K. Water stability of zeolite imidazolate framework 8 and application to porous membrane-protected micro-solid-phase extraction of polycyclic aromatic hydrocarbons from environmental water samples. J. Chromatogr. A 2011, 1218, 8490-8495. [CrossRef]

189. Song, X.; Li, J.; Xu, S.; Ying, R.; Ma, J.; Liao, C.; Liu, D.; Yu, J.; Chen, L. Determination of 16 polycyclic aromatic hydrocarbons in seawater using molecularly imprinted solid-phase extraction coupled with gas chromatography-mass spectrometry. Talanta 2012, 99, 75-82. [CrossRef]

190. Mauri-Aucejo, A.; Amorós, P.; Moragues, A.; Guillem, C.; Belenguer-Sapiña, C. Comparison of the solid-phase extraction efficiency of a bounded and an included cyclodextrin-silica microporous composite for polycyclic aromatic hydrocarbons determination in water samples. Talanta 2016, 32, 659-665. [CrossRef]

191. Soler-Seguí, S.; Belenguer-Sapiña, C.; Amorós, P.; Mauri-Aucejo, A. Evaluation of a cyclodextrin-silica hybrid microporous composite for the solid-phase extraction of polycyclic aromatic hydrocarbons. Anal. Sci. 2016, 32, 659-665. [CrossRef]

192. Wang, N.; Guo, Y.; Wang, L.; Liang, X.; Liu, S.; Jiang, S. Preparation of an aminopropyl imidazole-modified silica gel as a sorbent for solid-phase extraction of carboxylic acid compounds and polycyclic aromatic hydrocarbons. Analyst 2014, 139, 2531-2537. [CrossRef] [PubMed]

193. Zhao, W.; Yang, L.; He, L.; Zhang, S. Simultaneous Enrichment of Polycyclic Aromatic Hydrocarbons and $\mathrm{Cu}^{2+}$ in Water Using Tetraazacalix[2]arene[2]triazine as a Solid-Phase Extraction Selector. J. Agric. Food Chem. 2016, 64, 6233-6239. [CrossRef] [PubMed]

194. Kefi, B.B.; El Atrache, L.L.; Kochkar, H.; Ghorbel, A. $\mathrm{TiO}_{2}$ nanotubes as solid-phase extraction adsorbent for the determination of polycyclic aromatic hydrocarbons in environmental water samples. J. Environ. Sci. 2011, 23, 860-867. [CrossRef]

195. Huang, Y.; Zhou, Q.; Xie, G. Development of micro-solid phase extraction with titanate nanotube array modified by cetyltrimethylammonium bromide for sensitive determination of polycyclic aromatic hydrocarbons from environmental water samples. J. Hazard. Mater. 2011, 183, 82-89. [CrossRef] [PubMed]

196. Krupadam, R.J.; Korde, B.A.; Ashokkumar, M.; Kolev, S.D. Novel molecularly imprinted polymeric microspheres for preconcentration and preservation of polycyclic aromatic hydrocarbons from environmental samples. Anal. Bioanal. Chem. 2014, 406, 5313-5321. [CrossRef] [PubMed]

197. Foan, L.; Ricoul, F.; Vignoud, S. A novel microfluidic device for fast extraction of polycyclic aromatic hydrocarbons (PAHs) from environmental waters - comparison with stir-bar sorptive extraction (SBSE). Int. J. Environ. Anal. Chem. 2015, 95, 1171-1185. [CrossRef]

198. Wu, H.; Wang, X.; Liu, B.; Lu, J.; Du, B.; Zhang, L.; Ji, J.; Yue, Q.; Han, B. Flow injection solid-phase extraction using multi-walled carbon nanotubes packed micro-column for the determination of polycyclic aromatic hydrocarbons in water by gas chromatography-mass spectrometry. J. Chromatogr. A 2010, 1217, 2911-2917. [CrossRef]

199. Zhou, Y.Y.; Yan, X.P.; Kim, K.N.; Wang, S.W.; Liu, M.G. Exploration of coordination polymer as sorbent for flow injection solid-phase extraction on-line coupled with high-performance liquid chromatography for determination of polycyclic aromatic hydrocarbons in environmental materials. J. Chromatogr. A 2006, 1116, 172-178. [CrossRef]

200. Zhang, X.; Wang, P.; Han, Q.; Li, H.; Wang, T.; Ding, M. Metal-organic framework based in-syringe solid-phase extraction for the on-site sampling of polycyclic aromatic hydrocarbons from environmental water samples. J. Sep. Sci. 2018, 41, 1856-1863. [CrossRef]

(C) 2020 by the authors. Licensee MDPI, Basel, Switzerland. This article is an open access article distributed under the terms and conditions of the Creative Commons Attribution (CC BY) license (http://creativecommons.org/licenses/by/4.0/). 